\title{
Multicomponent Density-Functional Theory for Electrons and Nuclei
}

\author{
Thomas Kreibich \\ Institut für Theoretische Physik, Universität Würzburg, Am Hubland, D-97074 Würzburg, Germany \\ Robert van Leeuwen \\ Theoretical Chemistry, Materials Science Centre, University of Groningen, \\ Nijenborgh 4, 9747AG, Groningen, The Netherlands \\ E.K.U.Gross \\ Institut für Theoretische Physik, Freie Universität Berlin, Arnimallee 14, D-14195 Berlin, Germany
}

(Dated: August 24, 2018)

\begin{abstract}
We present a general multi-component density functional theory in which electrons and nuclei are treated completely quantum mechanically, without the use of a Born-Oppenheimer approximation. The two fundamental quantities in terms of which our theory is formulated are the nuclear N-body density and the electron density expressed in coordinates referring to the nuclear framework. For these two densities coupled Kohn-Sham equations are derived and the electron-nuclear correlation functional is analyzed in detail. The formalism is tested on the hydrogen molecule $\mathrm{H}_{2}$ and its positive ion $H_{2}^{+}$using several approximations for the electron-nuclear correlation functional.
\end{abstract}

\section{INTRODUCTION}

Density functional theory (DFT) is among the most succesful approaches to calculate the electronic structure of atoms, molecules and solids. In its original form [1, 2], DFT always invokes the Born-Oppenheimer approximation: One is supposed to calculate the electron density $\rho(\mathbf{r})$ which is in 1-1 correspondence to the static potential potential of fixed nuclei. In a recent Letter [3] we introduced a multicomponent density-functional theory (MCDFT) for the complete quantum treatment of manyparticle systems consisting of electrons and nuclei. With this theory it is possible to decribe from first principles physical phenomena that depend on a strong coupling between electronic and nuclear motion. MCDFT thereby extends the widely applied density functional formalism for purely electronic properties, opening up a new field of applications, such as the first-principles calculation of electron-phonon coupling in solids [4] which is a key ingredient in the description of superconductivity $5,6,6,8$ and polaronic motion 9, 10]. The quantum treatment of the nuclear motion in molecules or solids is essential in situations that from a Born-Oppenheimer (BO) viewpoint must be described by a superposition of different BO structures. This is, for instance, the case in floppy molecules [1] , or in so-called switchable molecules 12. which are in a superposition of an open and a closed state after a laser excitation. Apart from treating such various phenomena the MCDFT presented here also paves the way for future time-dependent extensions of the theory which would enable one to calculate the coupled electronic and nuclear dynamics of many-particle systems, within linear response and beyond. Indeed, some preliminary steps towards the description of the coupled ionization and dissociation dynamics of molecules in strong laser fields have already been taken [13, 14, 15].

The purpose of the present work is twofold. First, we want to give an extended and detailed description of the theory that was briefly described in our Letter. Second, we want to investigate in detail some new approximate density functionals for the electron-nuclear correlation and see how they perform. To do this the formalism is tested on the hydrogen molecule and its positive ion. The paper is organized as follows. In section II we first introduce the basic formalism and discuss the HohenbergKohn theorem and the Kohn-Sham equations in a multicomponent theory in which the electron density is defined with respect to a coordinate frame attached to the nuclear framework and in which the diagonal of the nuclear density matrix appears as a new variable. In section III we perform an analysis of the several energy functionals and of the resulting potentials in the Kohn-Sham equations. Furthermore, the connections between the effective potential of the nuclear Kohn-Sham equation and the Born-Oppenheimer energy surface is analyzed. In section IV we apply our formalism and test several approximate forms for the electron-nuclear correlation functional for the case of the hydrogen molecule and its positive ion. Finally in section $\mathrm{V}$ we present our conclusions.

\section{BASIC FORMALISM}

\section{A. Discussion of the Hamiltonian}

We consider a system composed of $N_{e}$ electrons with coordinates $\left\{\mathbf{r}_{j}\right\} \equiv \underline{\underline{\mathbf{r}}}$ and $N_{n}$ nuclei with masses $M_{1} \ldots M_{N_{n}}$, charges $Z_{1} \ldots Z_{N_{n}}$, and coordinates denoted by $\left\{\mathbf{R}_{\alpha}\right\} \equiv \underline{\underline{\mathbf{R}}}$. By convention, the subscript "e" and "n" refer to electrons and nuclei, respectively, and atomic units are employed throughout this work. In non-relativistic quantum mechanics, the system is decribed by the Hamiltonian

$$
\hat{H}=\hat{T}_{\mathrm{n}}(\underline{\underline{\mathbf{R}}})+\hat{W}_{n n}(\underline{\underline{\mathbf{R}}})+\hat{U}_{\mathrm{ext}, n}(\underline{\underline{\mathbf{R}}})
$$




$$
\begin{aligned}
& +\hat{T}_{e}(\underline{\underline{\mathbf{r}}})+\hat{W}_{e e}(\underline{\underline{\mathbf{r}}})+\hat{U}_{\text {ext }, e}(\underline{\underline{\mathbf{r}}}) \\
& +W_{e n}(\underline{\underline{\mathbf{R}}}, \underline{\underline{\mathbf{r}}})
\end{aligned}
$$

where

$$
\begin{aligned}
& \hat{T}_{n}=\sum_{\alpha=1}^{N_{n}}\left(-\frac{\nabla_{\alpha}^{2}}{2 M_{\alpha}}\right) \\
& \hat{T}_{e}=\sum_{j=1}^{N_{e}}\left(-\frac{\nabla_{j}^{2}}{2}\right)
\end{aligned}
$$

denote the kinetic-energy operators of the nuclei and electrons, respectively, and

$$
\begin{aligned}
\hat{W}_{n n} & =\frac{1}{2} \sum_{\substack{\alpha, \beta=1 \\
\alpha \neq \beta}}^{N_{n}} \frac{Z_{\alpha} Z_{\beta}}{\left|\mathbf{R}_{\alpha}-\mathbf{R}_{\beta}\right|} \\
\hat{W}_{e e} & =\frac{1}{2} \sum_{\substack{i, j=1 \\
i \neq j}}^{N_{e}} \frac{1}{\left|\mathbf{r}_{i}-\mathbf{r}_{j}\right|} \\
\hat{W}_{e n} & =-\sum_{j=1}^{N_{e}} \sum_{\alpha=1}^{N_{n}} \frac{Z_{\alpha}}{\left|\mathbf{r}_{j}-\mathbf{R}_{\alpha}\right|}
\end{aligned}
$$

represent the interparticle Coulomb interactions. We emphasize that no BO approximation has been assumed in (11); the Hamiltonian of Eq.(11) provides a quantum mechanical description of all, i.e., electronic and nuclear degrees of freedom. In contrast to the standard approach using the $\mathrm{BO}$ approximation, the interactions between electrons and nuclei are therefore treated within $\hat{W}_{e n}$, Eq. (6), and do not contribute to the external potentials. Truly external potentials representing, e.g., a voltage applied to the system, are contained in

$$
\begin{aligned}
& \hat{U}_{\text {ext }, n}=\sum_{\alpha=1}^{N_{n}} U_{\text {ext }, n}\left(\mathbf{R}_{\alpha}\right) \\
& \hat{U}_{\text {ext }, e}=\sum_{j=1}^{N_{e}} u_{\text {ext }, e}\left(\mathbf{r}_{j}\right) .
\end{aligned}
$$

Defining electronic and nuclear single-particle densities conjugated to the true external potential (7), a MCDFT formalism can readily be formulated on the basis of the above Hamiltonian [16]. However, as discussed in [3], such a MCDFT is not useful in practice because the single-particle densities necessarily reflect the symmetry of the true external potentials and are therefore not characteristic of the internal properties of the system. In particular, for all isolated systems where the external potentials (7) vanish, these densities, as a consequence of the translational invariance of the respective Hamiltonian, are constant.

A suitable MCDFT is obtained by defining the densities with respect to internal coordinates of the system [3]. To this end, new electronic coordinates are introduced according to

$$
\mathbf{r}_{j}^{\prime}=\mathcal{R}(\alpha, \beta, \gamma)\left(\mathbf{r}_{j}-\mathbf{R}_{\mathrm{CMN}}\right) \quad j=1 \ldots N_{e},
$$

where

$$
\mathbf{R}_{\mathrm{CMN}}:=\frac{1}{M_{\mathrm{nuc}}} \sum_{\alpha=1}^{N_{n}} M_{\alpha} \mathbf{R}_{\alpha} .
$$

denotes the center of mass (CM) of the nuclei, the total nuclear mass is given by

$$
M_{\mathrm{nuc}}=\sum_{\alpha=1}^{N_{n}} M_{\alpha},
$$

and $\mathcal{R}$ is the three-dimensional orthogonal matrix representing the Euler rotations 17]. The Euler angles $(\alpha, \beta, \gamma)$ are functions of the nuclear coordinates $\{\underline{\underline{\mathbf{R}}}\}$ and specify the orientation of the body-fixed coordinate frame. They can be determined in various ways. One way to define them is by requiring the inertial tensor of the nuclei to be diagonal in the body-fixed frame. The conditions that the off-diagonal elements of the inertia tensor are zero in terms of the rotated coordinates $\mathcal{R}\left(\mathbf{R}_{\alpha}-\mathbf{R}_{C M N}\right)$ then give three determining equations for the three Euler angles in terms of the nuclear coordinates $\{\underline{\mathbf{R}}\}$. This way of choosing the Euler angles is commonly used within the field of nuclear physics [18, 19, 20] but is, of course, not unique. A common alternative way to determine the orientation of the body-fixed system is provided by the so-called Eckart conditions [21, 22, 23, 24, 25, 26, 27] which are suitable to describe small vibrations in molecules and phonons in solids [4]. A general and very elegant discussion on the various ways the body-fixed frame can be chosen is given in reference [28]. In this work we will not make a specific choice as our derivations are independent of such a choice. The most important point is that by virtue of Eq. (9), the electronic coordinates are defined with respect to a coordinate frame that is attached to the nuclear framework and rotates as the nuclear framework rotates. In fact, this transformation comprises two transformations: A first one transforming the space-fixed inertial coordinates into $C M$-fixed relative coordinates, and a second one transforming the $C M$-fixed relative coordinates into body-fixed internal coordinates.

The nuclear coordinates themselves are not transformed any further at this point, i.e.,

$$
\mathbf{R}_{\alpha}^{\prime}=\mathbf{R}_{\alpha} \quad \alpha=1 \ldots N_{n} .
$$

Of course, introducing internal nuclear coordinates is also desirable. However, the choice of such coordinates depends strongly on the specific system to be described: If near-equilibrium situations in systems with well-defined geometries are considered, normal or - for a solid phonon coordinates are most appropriate, whereas fragmentation processes of molecules are better described in terms of Jacobi coordinates 29]. Therefore, keeping a high degree of flexibility, the nuclear coordinates are left unchanged for the time being and are only transformed to internal coordinates prior to actual applications in the 
final equations that we will derive. Another reason for not introducing any internal nuclear coordinates at this point, is to retain simple forms of the equations. In a transformation to internal nuclear coordinates typically the nuclear center-of-mass and the Euler angles are taken as new variables as well as $3 N_{n}-6$ internal or shape coordinates $Q_{i}[26,27,28]$. These internal coordinates, however, do not have a simple relation to the original $N_{n}$ nuclear coordinates and will therefore lead to a complicated form of the Hamiltonian in the new coordinates. We will therefore delay the use of such transformations until we have derived the final equations.

As a result of the coordinate changes of Eq.(9), the Hamiltonian (11) transforms into

$$
\begin{aligned}
\hat{H} & =\hat{T}_{n}(\underline{\underline{\mathbf{R}}})+\hat{W}_{n n}(\underline{\underline{\mathbf{R}}})+\hat{U}_{\mathrm{ext}, n}(\underline{\underline{\mathbf{R}}}) \\
& +\hat{T}_{e}\left(\underline{\underline{\mathbf{r}}}^{\prime}\right)+\hat{W}_{e e}\left(\underline{\underline{\mathbf{r}}}^{\prime}\right)+\hat{T}_{\mathrm{MPC}}\left(\underline{\underline{\mathbf{R}}}, \underline{\underline{\mathbf{r}}}^{\prime}\right) \\
& +\hat{W}_{e n}\left(\underline{\underline{\mathbf{R}}}, \underline{\underline{\mathbf{r}}}^{\prime}\right)+\hat{U}_{\text {ext }, e}\left(\underline{\underline{\mathbf{R}}}, \underline{\underline{\mathbf{r}}}^{\prime}\right) .
\end{aligned}
$$

Since we have transformed to a noninertial coordinate frame mass-polarization and Coriolis (MPC) terms

$$
\hat{T}_{\mathrm{MPC}}:=\sum_{\alpha=1}^{N_{n}}-\frac{1}{2 M_{\alpha}}\left(\nabla_{\mathbf{R}_{\alpha}}+\sum_{j=1}^{N_{e}} \frac{\partial \mathbf{r}_{j}^{\prime}}{\partial \mathbf{R}_{\alpha}} \nabla_{\mathbf{r}_{j}^{\prime}}\right)^{2}-\hat{T}_{n}(\underline{\underline{\mathbf{R}}})
$$

appear. Obviously, $\hat{T}_{\mathrm{MPC}}$ is not symmetric in the electronic and nuclear coordinates. However, this was not expected since only the electrons refer to a noninertial coordinate frame, whereas the nuclei are still defined with respect to the inertial frame. Therefore, all MPC terms arise solely from the electronic coordinates, representing ficticious forces due to the electronic motion in noninertial systems (for a detailed form of these terms within the current coordinate transformation see [4]). The kineticenergy operators $\hat{T}_{e}$ and $\hat{T}_{n}$, the electron-electron and nuclear-nuclear interactions, as well as the true external potential $\hat{U}_{\mathrm{ext}, n}$ acting on the nuclei are formally unchanged in Eq. (13) and therefore given by Eqs. (2) and (44) with the new coordinates replacing the old ones, whereas the electron-nuclear interaction now reads

$$
\begin{aligned}
& \hat{W}_{e n}\left(\underline{\underline{\mathbf{R}}}, \underline{\underline{\mathbf{r}}}^{\prime}\right) \\
& =-\sum_{j=1}^{N_{e}} \sum_{\alpha=1}^{N_{n}} \frac{Z_{\alpha}}{\left|\mathcal{R}(\alpha, \beta, \gamma)^{-1} \mathbf{r}_{j}^{\prime}-\mathbf{R}_{\alpha}+\mathbf{R}_{C M N}\right|} \\
& =-\sum_{j=1}^{N_{e}} \sum_{\alpha=1}^{N_{n}} \frac{Z_{\alpha}}{\left|\mathbf{r}_{j}^{\prime}-\mathcal{R}(\alpha, \beta, \gamma)\left(\mathbf{R}_{\alpha}-\mathbf{R}_{C M N}\right)\right|}
\end{aligned}
$$

The quantity

$$
\mathbf{R}_{\alpha}^{\prime \prime}=\mathcal{R}(\alpha, \beta, \gamma)\left(\mathbf{R}_{\alpha}-\mathbf{R}_{C M N}\right)
$$

that appears in Eq. (15) is a so-called shape coordinate [4, 28], i.e. it is invariant under rotations and translations of the nuclear framework

$$
\mathbf{R}_{\alpha}^{\prime \prime}(O \underline{\underline{\mathbf{R}}}+\mathbf{a})=\mathbf{R}_{\alpha}^{\prime \prime}(\underline{\underline{\mathbf{R}}})
$$

where $O$ is an arbitrary rotation matrix and a an arbitrary translation vector. The invariance property described in Eq. (17) is simply a consequence of the fact that the Euler angles are defined by giving the vectors $\mathbf{R}_{\alpha}^{\prime \prime}$ certain values, independent of where the nuclear centerof-mass was situated in the laboratory frame or how the nuclear framework was orientated. This is, of course, precisely the purpose of introducing a body-fixed frame. For this reason the potential in Eq. (15) that the electrons in the body-fixed frame experience from the nuclei is invariant under rotations or translations of the nuclear framework.

As a further result of the coordinate transformation (91), the true external potential acting on the electrons now not only depends on the electronic coordinates, but also on all the nuclear coordinates:

$$
\hat{U}_{\mathrm{ext}, e}\left(\underline{\underline{\mathbf{R}}}, \underline{\underline{r}}^{\prime}\right)=\sum_{j=1}^{N_{e}} u_{\mathrm{ext}, e}\left(\mathcal{R}^{-1} \mathbf{r}_{j}^{\prime}+\mathbf{R}_{C M N}\right) .
$$

In the chosen coordinate system the electron-nuclear interaction (15) and the external potential (18) remain onebody operators with respect to the electronic degrees of freedom but represent complicated $N_{n}$-body interactions with respect to the nuclei. We finally discuss some general aspects of our coordinate transformation. If we consider the symmetry properties of our original Hamiltonian of Eq. (11) in the absence of external potentials, we see that it is invariant under simultaneous translations and rotations of all particles, i.e. of both electrons and nuclei. This is not true anymore for our transformed Hamiltonian. Since we transformed the electronic coordinates to a body-fixed frame we find that in the absence of external potentials the transformed Hamiltonian of Eq. (13) is invariant under translations and rotations of nuclear coordinates only. The corresponding groundstate wavefunction, if it is nondegenerate, will have the same invariance.

Let us next consider the permutational symmetry. The ground state wavefunction of the original Hamiltonian of Eq.(11) is antisymmetric under the interchange of electronic space-spin coordinates and symmetric or antisymmetric under interchange of nuclear space-spin coordinates of nuclei of the same type, depending on whether they are bosons or fermions. The ground state wavefunction of the transformed Hamiltonian of Eq.(13) will also be antisymmetric with respect to the interchange of electronic space-spin coordinates. However, the symmetry properties with respect to the interchange of the nuclear space-spin coordinates depend on the conditions that we choose to determine the Euler angles. If we choose a determining constraint for the Euler angles that is symmetric in the interchange of particles of the same type, then the transformed wavefunction will retain the permutational symmetry properties of the original wavefunction. This is, for instance, the case if we determine the Euler angles by the requirement that the nuclear inertia tensor be diagonal. However, if we choose a nonsymmetric constraint, such as the Eckart conditions, then 
the transformed wavefunction will have more complicated transformation properties under the interchange of nuclear spin-space coordinates since the interchange of two nuclear coordinates will then also change the Euler angles (a detailed account on this topic is given in Ref.[27]). This can lead to practical complications but will not affect our general formalism.

We finally note that the coordinate transformation we presented here did not aim at a separation of the constants of motion of the system (even for the case of isolated systems). In contrast, the transformation (9) was chosen such that the new electronic coordinates reflect the internal symmetry of the system. We thus arrive at a Hamiltonian which naturally lends itself as a starting point for the formulation of a MCDFT, as will be shown in the subsequent sections.

\section{B. Definition of the Densities}

As a first step towards the formulation of a density functional theory, one has to define the densities which will serve as the fundamental variables of the theory. Although this seems to be rather straightforward and is normally not discussed at length, a careful definition of the densities is of crucial importance in the current context.

As already mentioned above, it is not useful to define electronic and nuclear single-particle densities in terms of the inertial coordinates $\mathbf{r}$ and $\mathbf{R}$, since such densities necessarily reflect the symmetry of the corresponding true external potentials, e.g., Galilean symmetry for vanishing external potentials. Therefore, such single-particle densities are not characteristic for the internal properties of the system under consideration.

We proceed with the definition of a suitable set of densities, which should fulfill the following requirements:

- They should be characteristic for the internal properties of the system; in particular, they should be meaningful in the limit of vanishing external potentials.

- The basic electronic variable should be a singleparticle quantity.

- The treatment of the nuclear degrees of freedom should allow for appropriate descriptions of situations as different as near-equilibrium properties of solids and fragmentation processes of molecules.

A set of densities which meets these requirements is given by

$$
\begin{aligned}
\Gamma(\underline{\underline{\mathbf{R}}}) & =\sum_{s, \sigma} \int d^{N_{e}} \mathbf{r}^{\prime}\left|\Psi\left(\underline{\underline{\mathbf{R}}}, \underline{\underline{\mathbf{r}^{\prime}}} \underline{\underline{\sigma}}\right)\right|^{2} \\
\rho\left(\mathbf{r}^{\prime}\right) & =N_{e} \sum_{s, \sigma} \int d^{N_{n}} \mathbf{R} \int d^{N_{e}-1} \mathbf{r}^{\prime}\left|\Psi\left(\underline{\underline{\mathbf{R} s}}, \underline{\underline{\mathbf{r}^{\prime}}} \underline{\underline{\sigma}}\right)\right|^{2}(20)
\end{aligned}
$$

where $\Psi\left(\underline{\underline{\mathbf{R} s}}, \underline{\mathbf{r}}^{\prime} \underline{\underline{\sigma}}\right)$ corresponds to the ground state of Hamiltonian (13) and where $\underline{\underline{s}}$ and $\underline{\underline{\sigma}}$ denote the nuclear and electronic spin coordinates. These densities are defined with respect to the transformed coordinates $\left\{\underline{\underline{\mathbf{R}}}, \underline{\underline{\mathbf{r}}}^{\prime}\right\}$. In particular, the electronic single-particle density $\rho\left(\mathbf{r}^{\prime}\right)$ refers to the body-fixed molecular frame. In terms these coordinates, the quantity (20) represents a conditional density, which is characteristic for the internal properties of the system. It is proportional to the probability density of finding an electron at position $\mathbf{r}^{\prime}$ as measured from the nuclear center-of-mass, given a certain orientation of the nuclear framework. Therefore the electronic density calculated through (20) reflects the internal symmetries of the system, e.g., the cylindrical symmetry of a diatomic molecule, instead of the Galilean symmetry of the underlying space. The nuclear degrees of freedom, on the other hand, are described using the diagonal of the nuclear density matrix, Eq. (19). In the absence of external potentials this quantity will have the transformation property

$$
\Gamma(O \underline{\underline{\mathbf{R}}}+\mathbf{a})=\Gamma(\underline{\underline{\mathbf{R}}})
$$

where $O$ is a rotation and a a translation vector. Its permutational properties will depend on the choice of the body fixed frame as discussed in the previous section. The quantity $\Gamma(\underline{\underline{\mathbf{R}}})$ allows us to set up a general as well as flexible formalism, which will be applicable to a large variety of situations. In an actual application, one may at a later stage further contract this quantity to obtain reduced density matrices or, depending on the physical situation, introduce more suitable internal nuclear coordinates which could not be done if single-particle quantities had already been introduced at this point.

\section{The Hohenberg-Kohn Theorem for Multicomponent Systems}

In this section, we discuss the extension of the Hohenberg-Kohn theorem to multicomponent systems. In contrast to prior formulations of the MCDFT 16, 30, 31, 32, 33], this analysis will employ the densities (19) and (20) as fundamental variables. Correspondingly, the starting point of the following analysis is the Hamiltonian (13). In order to formulate a Hohenberg-Kohn-(HK)type statement, the Hamiltonian (13) is generalized to

$$
\hat{H}=\hat{T}+\hat{W}+\hat{U}+\hat{V}
$$

where

$$
\hat{T}=\hat{T}_{n}(\underline{\underline{\mathbf{R}}})+\hat{T}_{e}\left(\underline{\underline{\mathbf{r}}}^{\prime}\right)+\hat{T}_{\mathrm{MPC}}\left(\underline{\underline{\mathbf{R}}}, \underline{\underline{\mathbf{r}}}^{\prime}\right)
$$

denotes the total kinetic-energy operator and

$$
\hat{W}=\hat{W}_{e e}\left(\underline{\underline{\mathbf{r}}}^{\prime}\right)+\hat{W}_{e n}\left(\underline{\underline{\mathbf{R}}}, \underline{\underline{\mathbf{r}}}^{\prime}\right)
$$

contains the electron-electron and the electron-nuclear interaction. Furthermore, auxiliary 'external' potentials 
conjugated to the densities (19) and (20),

$$
\hat{V}=\hat{V}_{n}(\underline{\underline{\mathbf{R}}})+\hat{V}_{e}\left(\underline{\underline{\mathbf{r}}}^{\prime}\right),
$$

have been added to the Hamiltonian. We note that, in the transformed coordinates, $\hat{V}_{n}$ actually acts as an $N_{n^{-}}$ body operator with respect to the nuclear coordinates,

$$
\hat{V}_{n}=V_{n}(\underline{\underline{\mathbf{R}}})
$$

and particularly contains the internuclear repulsion $\hat{W}_{n n}(\underline{\mathbf{R}})$, while $\hat{V}_{e}$ is a one-body operator with respect to the (body-fixed) electronic coordinates:

$$
\hat{V}_{e}=\sum_{j=1}^{N_{e}} v_{e}\left(\mathbf{r}_{j}^{\prime}\right) .
$$

The 'true' external potentials, on the other hand, are subsumed in

$$
\hat{U}=\hat{U}_{\text {ext }, n}(\underline{\underline{\mathbf{R}}})+\hat{U}_{\text {ext }, e}\left(\underline{\underline{\mathbf{R}}}, \underline{\underline{\mathbf{r}}}^{\prime}\right) .
$$

Note that the nuclear potential $\hat{U}_{\text {ext }, n}$ has the same structure as $\hat{V}_{n}$, whilst the electronic potential $\hat{U}_{\text {ext, } e}$ acts similar to the electron-nuclear interaction in the transformed coordinate system.

The Hamiltonian (22) and the above defined densities (19) and (20) now provide a suitable basis for the formulation of the multicomponent Hohenberg-Kohn (MCHK) theorem. It can be summarized by the following statements:

\section{Uniqueness:}

The set of ground-state densities $\{\Gamma, \rho\}$ uniquely determines the ground-state wavefunction, $\Psi=$ $\Psi[\Gamma, \rho], \quad$ as well as the potentials, $\left\{\hat{V}_{n}=\right.$ $\left.\hat{V}_{n}[\Gamma, \rho], \hat{V}_{e}=\hat{V}_{e}[\Gamma, \rho]\right\}$. As a consequence, any observable of the static many-body system is a functional of the set of ground-state densities $\{\Gamma, \rho\}$.

2. MCHK variational principle:

The total-energy functional

$$
E[\Gamma, \rho]:=\langle\Psi[\Gamma, \rho]|\hat{H}| \Psi[\Gamma, \rho]\rangle
$$

is equal to the exact ground-state energy $E_{0}$ if the exact densities $\Gamma_{0}$ and $\rho_{0}$ corresponding to fixed external potentials $\hat{V}_{n, 0}$ and $\hat{V}_{e, 0}$ are inserted into the functional. For all other densities, the inequality

$$
E_{0}<E[\Gamma, \rho]
$$

holds true.

This MCHK theorem can be proven by using both the reductio ad absurdum and the constrained search approach, familiar from standard DFT [34]. In the following, a generalization of the latter to multi-component (MC) systems will be presented. We start out by defining the functional:

$$
F[\Gamma, \rho]:=\min _{\Psi \rightarrow \Gamma, \rho}\langle\Psi|\hat{T}+\hat{W}+\hat{U}| \Psi\rangle,
$$

i.e., we search for the minimum of $\langle\Psi|\hat{T}+\hat{W}+\hat{U}| \Psi\rangle$ using all (properly normalized and symmetrized) wave functions yielding a given set of densities $\{\Gamma, \rho\}$. It must be noted that all the wave functions that we use in the constrained search procedure are now also required to have the correct symmetry properties respect to interchange of nuclear space-spin coordinates of nuclei of the same type. As we discussed before these symmetry properties depend on the way we define the body-fixed frame. For instance, if we define the body-fixed frame by a diagonalization of the nuclear inertia tensor then the constrained search must be carried out over all wavefunctions that are antisymmetric in the electronic spin-space coordinates and symmetric or anti-symmetric with respect to the interchange of nuclear spin-space coordinates, depending on whether the nuclei are bosons or fermions. If we denote the minimizing state (assuming it exists [59]) by $\Psi^{\min }[\Gamma, \rho]$, we realize that

$$
F[\Gamma, \rho]=\left\langle\Psi^{\min }[\Gamma, \rho]|\hat{T}+\hat{W}+\hat{U}| \Psi^{\min }[\Gamma, \rho]\right\rangle
$$

is - by construction - a functional of the densities. We note that, in contrast to usual DFT, the functional $F$ is not universal since it still depends on the external potentials $\hat{U}$ which, as a result of our coordinate transformation, are functions of both $\underline{\underline{\mathbf{R}}}$ and $\underline{\underline{\mathbf{r}}}^{\prime}$ as was discussed in connection with Eq. (18).

Using Eq.(32), the total-energy functional is given by

$$
E[\Gamma, \rho]=F[\Gamma, \rho]+\int d^{N_{n}} \mathbf{R} \Gamma(\underline{\underline{\mathbf{R}}}) V_{n}(\underline{\underline{\mathbf{R}}})+\int d \mathbf{r} \rho(\mathbf{r}) v_{e}(\mathbf{r}) .
$$

The variational principle (30) can now be proven by employing the Rayleigh-Ritz variational principle:

$$
E_{0}=\min _{\Psi}\langle\Psi|\hat{H}| \Psi\rangle
$$

Following the constrained-search procedure 35] of ordinary DFT, the minimum in (34) is split into two consecutive steps

$$
\begin{aligned}
E_{0}= & \min _{\Gamma, \rho}\left(\min _{\Psi \rightarrow \Gamma, \rho}\langle\Psi|\hat{H}| \Psi\rangle\right) \\
= & \min _{\Gamma, \rho}\left(F[\Gamma, \rho]+\int d^{N_{n}} \mathbf{R} \Gamma(\underline{\underline{\mathbf{R}}}) V_{n}(\underline{\underline{\mathbf{R}}})\right. \\
& \left.+\int d \mathbf{r} \rho(\mathbf{r}) v_{e}(\mathbf{r})\right) \\
= & \min _{\Gamma, \rho} E[\Gamma, \rho],
\end{aligned}
$$

where the external potentials $V_{n}$ and $v_{e}$ are held fixed during the minimization (For notational simplicity, the primes indicating the transformed coordinates are dropped from now on. By convention, all electronic coordinates are understood to refer to the body-fixed frame). In the second step, we have exploited the fact that all wave functions which lead to the same densities also yield the same external energy. By virtue of the RayleighRitz variational principle, the minimizing densities are 
the ground-state densities $\Gamma_{0}$ and $\rho_{0}$. Furthermore, any other set of densities will lead to an energy above the true ground-state energy if inserted in the total-energy functional (33). This completes the proof of statement 2.

In order to prove the first statement, we reformulate the variational principle (35) according to

$$
\delta\left\{F[\Gamma, \rho]+\int d^{N_{n}} \mathbf{R} \Gamma(\underline{\underline{\mathbf{R}}}) V_{n}(\underline{\underline{\mathbf{R}}})+\int d \mathbf{r} \rho(\mathbf{r}) v_{e}(\mathbf{r})\right\}=0 .
$$

Since the variations can be done independently, Eq. (36) is equivalent to

$$
\begin{gathered}
\frac{\delta F[\Gamma, \rho]}{\delta \Gamma(\underline{\underline{\mathbf{R}}})}+V_{n}(\underline{\underline{\mathbf{R}}})=0 \\
\frac{\delta F[\Gamma, \rho]}{\delta \rho(\mathbf{r})}+v_{e}(\mathbf{r})=0 .
\end{gathered}
$$

If the exact densities $\left\{\Gamma_{0}, \rho_{0}\right\}$ are inserted, the Euler equations (37) and (38) are satisfied for the true external potentials. If, on the other hand, an arbitrary set of densities $\{\Gamma, \rho\}$ is inserted, Eqs. (37) and (38) define - assuming the functional derivatives exist - a set of potentials, which reproduce $\{\Gamma, \rho\}$ as ground-state densities. Therefore, the set of densities $\{\Gamma, \rho\}$ uniquely determines the external potentials $\left\{V_{n}, v_{e}\right\}$ and thus the ground-state wavefunction $\Psi=\Psi^{\min }[\Gamma, \rho]$.

Before concluding, a number of remarks are added:

- As usual, the potentials are uniquely determined up to an arbitrary additive constant, and nondegeneracy of the ground state has been assumed.

- Similar to purely electronic DFT, the functional $F[\Gamma, \rho]$ is defined via Eq. (32) for all $\{\Gamma, \rho\}$ representable densities, i.e. for all densities obtained according to Eqs.(19) and (20) from a manybody wave function with the right permutational symmetries. The potentials $\left\{V_{n}, v_{e}\right\}$ are defined for all densities, for which the functional derivatives in Eqs. (37) and (38) exist, i.e., for all interacting $\left\{V_{n}, v_{e}\right\}$-representable densities.

- If vanishing external potentials (18) are considered, the analysis reduces to the one given in [3].

\section{The Kohn-Sham Scheme for Multicomponent Systems}

As usual, the HK theorem does not depend on the specific form of the particle-particle interaction. In particular, it can be applied to an auxiliary system which is characterized by $\hat{W}=0$, i.e., the system consists of noninteracting electrons and of nuclei that only interact amongst themselves. The key assumption in establishing the MCKS scheme is that local effective potentials $\left\{\hat{V}_{S, n}, \hat{V}_{S, e}\right\}$ exist such that the ground-state densities of the auxiliary system reproduce the exact ground-state densities $\left\{\Gamma_{0}, \rho_{0}\right\}$ of the fully interacting system. If that assumption holds true, the exact ground-state densities are given by

$$
\begin{gathered}
\Gamma_{0}(\underline{\underline{\mathbf{R}}})=\sum_{s}|\chi(\underline{\underline{\mathbf{R} s}})|^{2} \\
\rho_{0}(\mathbf{r})=\sum_{j=1}^{N_{e}}\left|\varphi_{j}(\mathbf{r})\right|^{2},
\end{gathered}
$$

where $\chi$ and $\varphi_{j}$ are solutions of an $N_{n}$-particle nuclear and a single-particle electronic Schrödinger equation, respectively:

$$
\begin{aligned}
\left(-\sum_{\alpha} \frac{\nabla_{\alpha}^{2}}{2 M_{\alpha}}+V_{S, n}(\underline{\underline{\mathbf{R}}})-\epsilon_{n}\right) \chi(\underline{\underline{\mathbf{R} s}}) & =0 \\
\left(-\frac{\nabla^{2}}{2}+v_{S, e}(\mathbf{r})-\epsilon_{e, j}\right) \varphi_{j}(\mathbf{r}) & =0 .
\end{aligned}
$$

By virtue of the MCHK theorem applied to the auxiliary system, the effective potentials $V_{S, n}(\underline{\underline{\mathbf{R}}})$ and $v_{S, e}(\mathbf{r})$ are uniquely determined by the ground-state densities $\left\{\Gamma_{0}, \rho_{0}\right\}$, once their existence is assumed. They are given by

$$
\begin{aligned}
V_{S, n}(\underline{\underline{\mathbf{R}}}) & =W_{n n}(\underline{\underline{\mathbf{R}}})+\left.\frac{\delta E_{\mathrm{U}, \mathrm{Hxc}}[\Gamma, \rho]}{\delta \Gamma(\underline{\underline{\mathbf{R}}})}\right|_{\Gamma_{0}, \rho_{0}} \\
v_{S, e}(\mathbf{r}) & =\left.\frac{\delta E_{\mathrm{U}, \mathrm{Hxc}}[\Gamma, \rho]}{\delta \rho(\mathbf{r})}\right|_{\Gamma_{0}, \rho_{0}} .
\end{aligned}
$$

In this procedure we require the nuclear wavefunction $\chi$ to have the same symmetry-properties under the interchange of nuclei of the same type as the exact wavefunction of the interacting system (this will also be required along the adiabatic connection to be discussed later in the paper). The last terms on the right-hand sides of Eqs. (43) and (44) represent the potentials due to all non-trivial interactions of the system, i.e., they contain the Hartree-exchange-correlation (Hxc) effects of the electron-electron and electron-nuclear interactions as well as mass-polarization and Coriolis effects and the influence of the true external potentials $\hat{U}$. As seen in Eqs. (43) and (44), these potentials are given as functional derivatives of the UHxc energy functional defined by

$$
E_{\mathrm{U}, \mathrm{Hxc}}[\Gamma, \rho]:=F[\Gamma, \rho]-T_{S, n}[\Gamma]-T_{S, e}[\rho] .
$$

This quantity represents the central quantity of the MCDFT and contains all many-body effects except the purely nuclear correlations. We note that, in the case of vanishing external potentials $\hat{U} \equiv 0$, the nuclear effective potentials $V_{S, n}(\underline{\underline{\mathbf{R}}})$ and the conjugated density, i.e., the nuclear density matrix $\Gamma(\underline{\underline{\mathbf{R}}})$ are invariant under translations. Therefore, the nuclear center-of-mass can be separated off in Eq. (41), reducing the number of degrees of freedom by three. We will illustrate this procedure in our applications later. 
In order to derive the above representations of the effective potentials, we consider the energy functional of the auxiliary system introduced above:

$$
\begin{aligned}
E_{S}[\Gamma, \rho] & =T_{S, n}[\Gamma]+T_{S, e}[\rho]+\int d^{N_{n}} \mathbf{R} \Gamma(\underline{\underline{\mathbf{R}}}) V_{S, n}(\underline{\underline{\mathbf{R}}}) \\
& +\int d \mathbf{r} \rho(\mathbf{r}) v_{S, e}(\mathbf{r}) .
\end{aligned}
$$

As noted before, the nuclear-nuclear interaction $\hat{W}_{n n}$ is included in the 'external' potential $V_{S, n}(\underline{\underline{\mathbf{R}}})$. The noninteracting kinetic-energy functional $T_{S, e}[\bar{\rho}]$ is the one familiar from purely electronic DFT,

$$
T_{S, e}[\rho]=\min _{\Phi \rightarrow \rho}\left\langle\Phi\left|\hat{T}_{e}\right| \Phi\right\rangle,
$$

where the minimization is over all electronic Slater determinants $\Phi$ yielding $\rho$. Similarly, the nuclear kineticenergy functional is given by

$$
T_{S, n}[\Gamma]=\min _{\chi \rightarrow \Gamma}\left\langle\chi\left|\hat{T}_{n}\right| \chi\right\rangle .
$$

In contrast to the electronic wavefunction $\Phi$, the nuclear wavefunction $\chi$ is not a Slater determinant, but a correlated many-body wavefunction, since it minimizes $\hat{T}_{n}$ under the constraint of generating the diagonal of the nuclear $N_{n}$-particle density matrix. We note that, although $\chi$ is an interacting many-body wavefunction, $T_{S, n}$ is not the interacting nuclear kinetic-energy functional $T_{n}[\Gamma, \rho]=\left\langle\Psi[\Gamma, \rho]\left|\hat{T}_{n}\right| \Psi[\Gamma, \rho]\right\rangle$, since $\Psi[\Gamma, \rho]$ minimizes $\langle\Psi|\hat{T}+\hat{W}+\hat{U}| \Psi\rangle$ (for given densities $\{\Gamma, \rho\}$ ), therefore including all electron-nuclei interactions as well as mass-polarization and Coriolis couplings. Assuming the densities $\{\Gamma, \rho\}$ to be noninteracting $\left\{V_{n}, v_{e}\right\}$ representable, the minimizing states of (47) and (48), i.e., the states minimizing the kinetic energy for given $\{\Gamma, \rho\}$, are obtained from Eqs. (41) and (42) with the potentials uniquely determined by the Euler equations following from (46):

$$
\begin{gathered}
\left.\frac{\delta T_{S, n}[\tilde{\Gamma}]}{\delta \tilde{\Gamma}(\underline{\underline{\mathbf{R}}})}\right|_{\Gamma}+V_{S, n}(\underline{\underline{\mathbf{R}}})=0 \\
\left.\frac{\delta T_{S, e}[\tilde{\rho}]}{\delta \tilde{\rho}(\mathbf{r})}\right|_{\rho}+v_{S, e}(\mathbf{r})=0
\end{gathered}
$$

Returning to the interacting problem, we decompose the functional $F[\Gamma, \rho]$ according to Eq. (45). Employing this definition in the variational equations (37) and (38) of the interacting problem and comparing them to the Euler equations (49) and (50), we find that the effective potentials which reproduce the exact densities from the auxiliary system are indeed given by Eqs. (43) and (44).

Eqs. (39)-(42), (43) and (44) constitute the MCKS system. Since the effective potentials depend on both densities, the MCKS equations (41) and (42) are coupled, reflecting the mutual influence of electrons and nuclei on each other, and have to be solved self-consistently. We emphasize that Eq. (42), although similar to the usual electronic KS equation, does not parametrically depend on the nuclear configuration. Instead, the information on the nuclear distribution is already included through the functional dependence on $\Gamma$. Considering the nuclear MCKS equation (41), we again realize its similarity with the conventional nuclear BO equation. Yet, no BO approximation has been used to derive Eq. (41). In contrast, since the MCKS scheme provides the exact ground state, all non-BO effects are, in principle, included. Whether or not the non-BO effects are reproduced in practical applications depends, of course, on the quality of the approximations employed for $E_{\mathrm{U}, \mathrm{Hxc}}[\Gamma, \rho]$. We also note that in the absence of external potentials the potential $V_{S, n}(\underline{\mathbf{R}})$ has the same symmetry properties as the BO-energy surface under rotations and translations, i.e.

$$
V_{S, n}(O \underline{\underline{\mathbf{R}}}+\mathbf{a})=V_{S, n}(\underline{\underline{\mathbf{R}}})
$$

The way it will transform under interchange of like nuclei will depend on the way we choose the body-fixed frame. It is also important to realize that when solving the nuclear equation (41) we must look for the solution $\chi(\underline{\underline{\mathbf{R} s}})$ with the lowest energy under the constraint that it has the correct symmetry under interchange of nuclear spacespin coordinates, i.e. the symmetry that was imposed by the constrained-search. Like the nuclear BO equation, the nuclear equation (41) is still a many-body equation. Therefore, its solution will, in general, be rather complicated and further simplifications are highly desirable. Typically, one first splits off the nuclear center-of-mass motion and the global rotations of the molecule. Then the remaining nuclear degrees of freedom are transformed to normal coordinates, in terms of which the problem is treated in a harmonic approximation, possibly including anharmonic effects in a mean-field fashion [36]. However, due to the generality of the method, different treatments appropriate for different physical situations can be used.

\section{ANALYSIS OF THE FUNCTIONALS}

\section{A. Decomposition of the Energy Functional}

In the last section, the foundations of the MCDFT were developed. We derived a formally exact scheme, which provides a way to calculate ground-state properties of MC systems. For any practical application, the functional $E_{\mathrm{U}, \mathrm{Hxc}}$ needs to be approximated. In order to gain more insight in the construction of such an approximation, this section discusses a number of rigorous properties of this functional.

Following [32, we start out by decomposing the UHxc energy functional (45) in parts associated with its various interactions. To this end, we define the following quantities:

$$
F^{e}[\rho]:=\min _{\psi \rightarrow \rho}\left\langle\psi\left|\hat{T}_{e}+\hat{W}_{e e}\right| \psi\right\rangle
$$




$$
\begin{aligned}
F^{e n}[\Gamma, \rho]:= & \min _{\psi \rightarrow \Gamma, \rho}\left\langle\psi\left|\hat{T}_{n}+\hat{T}_{e}+\hat{W}\right| \psi\right\rangle \\
T_{\mathrm{MPC}}[\Gamma, \rho]:= & \min _{\psi \rightarrow \Gamma, \rho}\left\langle\psi\left|\hat{T}_{n}+\hat{T}_{e}+\hat{T}_{\mathrm{MPC}}+\hat{W}\right| \psi\right\rangle \\
& -F^{e n}[\Gamma, \rho] \\
U_{\mathrm{Hxc}}[\Gamma, \rho]:= & F[\Gamma, \rho]-\min _{\psi \rightarrow \Gamma, \rho}\langle\psi|\hat{T}+\hat{W}| \psi\rangle .
\end{aligned}
$$

The first term represents the electronic functional which, by construction, is identical to the functional $F_{L L}[\rho]$ of standard electronic DFT, first introduced in [35]. Usually, this quantity is split according to

$$
F^{e}[\rho]=T_{S, e}[\rho]+E_{\mathrm{H}}^{e}[\rho]+E_{\mathrm{xc}}^{e}[\rho]
$$

where $E_{\mathrm{H}}^{e}$ is the electronic Hartree functional

$$
E_{\mathrm{H}}^{e}[\rho]:=\frac{1}{2} \int d \mathbf{r} d \mathbf{r}^{\prime} \frac{\rho(\mathbf{r}) \rho\left(\mathbf{r}^{\prime}\right)}{\left|\mathbf{r}-\mathbf{r}^{\prime}\right|}
$$

and where the electronic exchange-correlation functional $E_{\mathrm{xc}}^{e}$ is defined by Eq. (56). In contrast to $F^{e}$, the second functional $F^{e n}$ also includes the nuclear kinetic energy as well as the electron-nuclear interaction, but still neglects mass-polarization and Coriolis effects and the influence of the external potential $\hat{U}$. As discussed later on, the functional $F^{e n}$ thus includes in particular the effects arising from the electron-nuclear correlation. The first term on the right-hand side of Eq. (54) additionally contains the mass-polarization and Coriolis terms of the kinetic-energy operator. Therefore, the difference between $\min \langle\psi|\hat{T}+\hat{W}| \psi\rangle$ and $F^{e n}$ is responsible for mass-polarization and Coriolis effects and thus denoted by $T_{\mathrm{MPC}}$. Similarily, the last term denoted by $U_{\mathrm{Hxc}}$ takes care of all effects introduced by the true external potentials $\hat{U}$. Consequently, if no true external fields are applied to the system, $U_{\mathrm{Hxc}}$ vanishes identically.

Inserting Eqs. (52)-(55) into Eq. (45) leads to

$$
\begin{aligned}
E_{\mathrm{U}, \mathrm{Hxc}}[\Gamma, \rho] & =E_{\mathrm{H}}^{e}[\rho]+E_{\mathrm{xc}}^{e}[\rho]+E_{\mathrm{Hc}}^{e n}[\Gamma, \rho] \\
& +T_{\mathrm{MPC}}[\Gamma, \rho]+U_{\mathrm{Hxc}}[\Gamma, \rho],
\end{aligned}
$$

where

$$
E_{\mathrm{Hc}}^{e n}[\Gamma, \rho]:=F^{e n}[\Gamma, \rho]-T_{S, n}[\Gamma]-F^{e}[\rho] .
$$

Eq. (58) provides a decomposition of the Hxc energy functional in its natural contributions. The first part, given by $E_{\mathrm{H}}^{e}$ and $E_{\mathrm{xc}}^{e}$, describes the Coulomb interactions among the electrons. It is important to note that these functionals are, by construction, identical to the ones familiar from standard electronic DFT. The electronelectron interaction can therefore be treated in the familiar way, namely by using the widely investigated and highly successful approximations for the electronic xc energy functional $E_{\mathrm{xc}}^{e}[\rho]$. The last term of Eq. (58) was constructed to incorporate all effects arising from the presence of true external potentials $\hat{U}$. As already mentioned above, these terms are not of a single particle form in the transformed coordinate system and have to be treated similarly to the interaction terms. The functional $U_{\mathrm{Hxc}}[\Gamma, \rho]$ provides a means of dealing with these effects. Similarly, the fourth term of Eq. (58) incorporates all effects due to the mass-polarization and Coriolis terms. At least for ground-state properties, this term is expected to be unimportant and can be neglected in most situations. If such effects are, on the other hand, important in a given physical situation, they can, in principle, be included in the calculation by taking the functional $T_{\mathrm{MPC}}[\Gamma, \rho]$ explicitly into account. Finally, the term $E_{\mathrm{Hc}}^{e n}[\Gamma, \rho]$ contains all effects due to the electron-nuclear interaction. Its analysis will be continued in the next section.

The decomposition (58) of the energy functional $E_{\mathrm{U}, \mathrm{Hxc}}$ is obviously not unique. However, the charme of the above prescription lies in the fact that, firstly, parts like the purely electronic functionals are already well known such that one can rely on existing approximations for these functional. Secondly, the functionals (53)-(55) contain, by their very construction, just the effect of one specifically chosen interaction. This, in particular, guarantees that the functionals $E_{\mathrm{Hxc}}^{e}[\rho], E_{\mathrm{Hc}}^{e n}[\Gamma, \rho]$ and $T_{\mathrm{MPC}}[\Gamma, \rho]$ are universal in the sense that they do not depend on the external potentials and can therefore be employed for all systems independent of the applied external fields. All effects arising from the external potentials are subsumed in $U_{\mathrm{Hxc}}[\Gamma, \rho]$.

\section{B. The electron-nuclear energy functional $E_{\mathrm{Hc}}^{e n}$}

Using the decomposition (58), the well-studied electronic Hxc energy functional as well as the - at least for ground-state properties - presumably negligible masspolarization and Coriolis contribution were separated off in the functional $E_{\mathrm{U}, \mathrm{Hxc}}$. In this section, we discuss the functional $E_{\mathrm{Hc}}^{e n}[\Gamma, \rho]$ which contains the many-body effects due to the electron-nuclear interaction. We will derive an equation for this functional that is of a suitable form to be used in our approximations later. To do this we will use the familiar coupling constant integration technique of standard density-functional theory. To begin with, we consider the Hamiltonian

$$
\hat{H}_{\lambda}=\hat{T}_{n}+\hat{T}_{e}+\hat{W}_{e e}+\lambda \hat{W}_{e n}+\hat{V}_{n, \lambda}+\hat{V}_{e, \lambda},
$$

where a non-negative coupling constant $\lambda$, scaling the electron-nuclear interaction, has been introduced. As usual, the potential $\hat{V}_{\lambda}=\hat{V}_{n, \lambda}+\hat{V}_{e, \lambda}$ is chosen such that the densities remain fixed: $\Gamma_{\lambda}=\Gamma$ and $\rho_{\lambda}=\rho$, independent of the coupling constant $\lambda$. Employing the couplingconstant integration technique 37, 38, 39] adapted to the electron-nuclear interaction [32], the electron-nuclear Hc energy functional (59) is rewritten as

$$
\begin{aligned}
& E_{\mathrm{Hc}}^{e n}[\Gamma, \rho] \\
& =\left.\min _{\Psi^{\lambda} \rightarrow \Gamma, \rho}\left\langle\Psi^{\lambda}\left|\hat{T}_{n}+\hat{T}_{e}+\hat{W}_{e e}+\lambda \hat{W}_{e n}\right| \Psi^{\lambda}\right\rangle\right|_{\lambda=1} \\
& \quad-\left.\min _{\Psi^{\lambda} \rightarrow \Gamma, \rho}\left\langle\Psi^{\lambda}\left|\hat{T}_{n}+\hat{T}_{e}+\hat{W}_{e e}+\lambda \hat{W}_{e n}\right| \Psi^{\lambda}\right\rangle\right|_{\lambda=0}
\end{aligned}
$$




$$
\begin{aligned}
& =\int_{0}^{1} d \lambda \frac{\partial}{\partial \lambda}\left\langle\Psi_{\Gamma, \rho}^{\min , \lambda}\left|\hat{T}_{n}+\hat{T}_{e}+\hat{W}_{e e}+\lambda \hat{W}_{e n}\right| \Psi_{\Gamma, \rho}^{\min , \lambda}\right\rangle \\
& =\int_{0}^{1} d \lambda\left\langle\Psi_{\Gamma, \rho}^{\min , \lambda}\left|\hat{W}_{e n}\right| \Psi_{\Gamma, \rho}^{\min , \lambda}\right\rangle,
\end{aligned}
$$

where $\Psi_{\Gamma, \rho}^{\min , \lambda}$ denotes the minimizing state of $\left\langle\hat{T}_{n}+\hat{T}_{e}+\right.$ $\left.\hat{W}_{e e}+\lambda \hat{W}_{e n}\right\rangle$ generating the given densities $\{\Gamma, \rho\}$, and a Hellmann-Feynman-type theorem was used in the last step. Therefore, the electron-nuclear energy functional $E_{\mathrm{Hc}}^{e n}$ is given by

$$
\begin{aligned}
& E_{\mathrm{Hc}}^{e n}[\Gamma, \rho] \\
& \left.=\int d^{N_{n}} \mathbf{R} \Gamma \underline{\underline{\mathbf{R}}}\right) \int d \mathbf{r} W_{e n}(\underline{\underline{\mathbf{R}}}, \mathbf{r}) \bar{\gamma}^{\min }[\Gamma, \rho](\mathbf{r} \mid \underline{\underline{\mathbf{R}}}),
\end{aligned}
$$

where

$$
\begin{aligned}
W_{e n}(\underline{\underline{\mathbf{R}}}, \mathbf{r}) & =-\sum_{\alpha} \frac{Z_{\alpha}}{\left|\mathcal{R}^{-1} \mathbf{r}-\mathbf{R}_{\alpha}+\mathbf{R}_{C M N}\right|} \\
& =-\sum_{\alpha} \frac{Z_{\alpha}}{\left|\mathbf{r}-\mathcal{R}\left(\mathbf{R}_{\alpha}-\mathbf{R}_{C M N}\right)\right|} .
\end{aligned}
$$

The electronic conditional density $\gamma$ is defined by

$$
\gamma(\mathbf{r} \mid \underline{\underline{\mathbf{R}}}):=N_{e} \sum_{\sigma, s} \int d^{N_{e}-1} \mathbf{r}|\Psi(\underline{\underline{\mathbf{r}} \sigma}, \underline{\underline{\mathbf{R} s}})|^{2} / \Gamma(\underline{\underline{\mathbf{R}}}),
$$

and $\bar{\gamma}$ represents the coupling-constant average of $\gamma$. The conditional density satisfies important sumrules that we will use later for the construction of approximate functionals:

$$
\begin{aligned}
N_{e} & =\int d \mathbf{r} \gamma(\mathbf{r} \mid \underline{\underline{\mathbf{R}}}) \quad \forall \underline{\underline{\mathbf{R}}} \\
\rho(\mathbf{r}) & =\int d \underline{\underline{\mathbf{R}}} \Gamma(\underline{\underline{\mathbf{R}}}) \gamma(\mathbf{r} \mid \underline{\underline{\mathbf{R}}})
\end{aligned}
$$

By virtue of Eq. (62), the Hc energy can be interpreted as the electrostatic interaction energy of the (couplingconstant averaged) electronic density for a fixed nuclear configuration with the point charges of the corresponding nuclei, averaged over the nuclear distribution. This interpretation will play an important role in our later development of approximate functionals.

In order to gain further insight into the electronnuclear Hc energy functional, we now establish a connection between the MCDFT scheme and the conventional BO method which provides a highly successful treatment of electron-nuclear correlation. To that end, we decompose the total wavefunction into an adiabatic product according to

$$
\Psi_{\Gamma, \rho}^{\min }(\underline{\underline{\mathbf{r} \sigma}}, \underline{\underline{\mathbf{R} s}})=\chi(\underline{\underline{\mathbf{R} s}}) \Xi[\Gamma, \rho](\underline{\underline{\mathbf{r} \sigma}} \underline{\underline{\mathbf{R} s}}),
$$

where $\chi$ is the nuclear wavefunction generating the nuclear density matrix $\Gamma$ and $\Xi[\Gamma, \rho]$ is an electronic state normalized to one for every nuclear configuration $\underline{\underline{\mathbf{R} s}}$ :

$$
\sum_{\sigma} \int d^{N_{e}} \mathbf{r}|\Xi[\Gamma, \rho](\underline{\underline{\mathbf{r} \sigma}} \mid \underline{\underline{\mathbf{R} s}})|^{2}=1
$$

We note that the decomposition (67) is actually an exact representation of the correlated electron-nuclear wave function 40, 41, 42, 43, 44, 45 and that the factors $\chi$ and $\Xi$ are unique [46] up to within an $\underline{\underline{\mathbf{R} s} \text {-dependent }}$ phasefactor. However, it is important to note that the electronic state $\Xi$ is not identical to the usual electronic $\mathrm{BO}$ state. Even if non-BO effects were neglected, $\Xi$ would not be identical to the electronic BO state since $\chi$ and $\Xi$ are required to reproduce a given set of densities $(\Gamma, \rho)$ (the two electronic wavefunctions only become equivalent, if $\Xi[\Gamma, \rho]$ is evaluated at the $\mathrm{BO}$ densities $\left.(\Gamma, \rho)=\left(\Gamma^{\mathrm{BO}}, \rho^{\mathrm{BO}}\right)\right)$. Instead, $\Xi[\Gamma, \rho]$ is expanded according to

$$
\Xi[\Gamma, \rho](\underline{\underline{\mathbf{r} \sigma}} \underline{\underline{\mathbf{R} s}})=\sum_{k} a_{k}[\Gamma, \rho](\underline{\underline{\mathbf{R} s}}) \Xi_{\underline{\underline{\mathbf{R}}, k}}^{\mathrm{BO}}(\underline{\underline{\mathbf{r} \sigma}}),
$$

where $\left\{\Xi_{\underline{\underline{\mathbf{R}}}, k}^{\mathrm{BO}}\right\}$ denotes a complete set of (BO) eigenfunctions corresponding to the electronic (clamped-nuclei) Hamiltonian $\hat{H}_{e}:=\hat{T}_{e}+\hat{W}_{e e}+\hat{W}_{e n}$. Employing Eq. (67) together with (68) and assuming that $\Xi[\Gamma, \rho]$ is real, the electron-nuclear Hc energy functional is given from Eq. (59) by

$$
\begin{aligned}
& E_{\mathrm{Hc}}^{e n}[\Gamma, \rho] \\
& =\left\langle\Psi_{\Gamma, \rho}^{\min }\left|\hat{T}_{n}+\hat{H}_{e}\right| \Psi_{\Gamma, \rho}^{\min }\right\rangle-T_{S, n}[\Gamma]-F^{e}[\rho] \\
& =\sum_{s} \int d^{N_{n}} \mathbf{R}|\chi(\underline{\underline{\mathbf{R} s}})|^{2}\left\langle\Xi[\Gamma, \rho]\left|\hat{T}_{n}+\hat{H}_{e}\right| \Xi[\Gamma, \rho]\right\rangle_{e}-F^{e}[\rho]
\end{aligned}
$$

where the index "e" at the bracket indicates that the integration is over electronic coordinates only. Using Eq.(69) we then obtain

$$
\begin{aligned}
& E_{\mathrm{Hc}}^{e n}[\Gamma, \rho] \\
& =\left.\sum_{s} \int d^{N_{n}} \mathbf{R}|\chi(\underline{\underline{\mathbf{R} s}})|^{2} \sum_{k, l}\left\{\mid a_{k}[\Gamma, \rho] \underline{\underline{\mathbf{R} s}}\right)\right|^{2} \cdot \epsilon_{k}^{\mathrm{BO}}(\underline{\underline{\mathbf{R}}}) \delta_{k, l} \\
& \left.\left.+a_{k}^{\star}[\Gamma, \rho] \underline{\underline{\mathbf{R} s}}\right)\left\langle\Xi_{k}^{\mathrm{BO}}\left|\hat{T}_{n}\right| \Xi_{l}^{\mathrm{BO}}\right\rangle_{e} a_{l}[\Gamma, \rho](\underline{\underline{\mathbf{R} s}})\right\}-F^{e}[\rho],
\end{aligned}
$$

where

$$
\epsilon_{k}^{\mathrm{BO}}(\underline{\underline{\mathbf{R}}})=\left\langle\Xi_{k}^{\mathrm{BO}}\left|\hat{H}_{e}\right| \Xi_{k}^{\mathrm{BO}}\right\rangle_{e}
$$

represents the $k$ th $\mathrm{BO}$ potential-energy surface (PES) and the index "e" at the bracket indicates that the integration is over electronic coordinates only. On the basis of Eq. (71), one can interpret $E_{\mathrm{Hc}}^{e n}$ as the potential energy of the nuclei, where the nuclear distribution lies in a potential hypersurface, which is composed of adiabatic BO-PES weighted with the coefficients $a_{k}[\Gamma, \rho]$ as well as nonadiabatic corrections to it. Of course, the coefficients $a_{k}$ and their functional dependence on the set of densities $[\Gamma, \rho]$ is unknown at this point. However, Eq. (71) helps us in gaining a better understanding of the electron-nuclear Hc energy functional and establishes an - at least - formal link to the BO scheme which is further exploited when the effective potentials are discussed later on. 


\section{Concerning the true external potentials}

Similar to the techniques employed in the last section, the coupling-constant integration can be employed to derive an expression for the functional $U_{\mathrm{Hxc}}[\Gamma, \rho]$ which subsumes the many-body effects arising from the true external field. In analogy to Eq. (62), one obtains

$$
\begin{aligned}
& U_{\mathrm{Hxc}}[\Gamma, \rho] \\
& =\int d^{N_{n}} \mathbf{R} \Gamma(\underline{\underline{\mathbf{R}}}) \int d \mathbf{r} U_{\text {ext }}(\underline{\underline{\mathbf{R}}}, \mathbf{r}) \bar{\gamma}^{\min ^{\prime}}[\Gamma, \rho](\mathbf{r} \underline{\underline{\mathbf{R}}}),
\end{aligned}
$$

where $\bar{\gamma}^{\min ^{\prime}}$ again denotes the coupling-constant average with respect to coupling constant $\mu$ of the conditional density $\gamma^{\min ^{\prime}, \mu}[\Gamma, \rho]$ corresponding to the states $\Psi_{\Gamma, \rho}^{\min ^{\prime}, \mu}$ minimizing $\langle\hat{T}+\hat{W}+\mu \hat{U}\rangle$. We further defined $U_{\text {ext }}(\underline{\underline{\mathbf{R}}}, \mathbf{r})$ to be

$$
U_{\text {ext }}(\underline{\underline{\mathbf{R}}}, \mathbf{r}):=\frac{1}{N_{e}} U_{\text {ext }, n}(\underline{\underline{\mathbf{R}}})+u_{\mathrm{ext}, e}\left(\mathcal{R}^{-1} \mathbf{r}+\mathbf{R}_{C M N}\right)
$$

It has to be noted that the conditional densities appearing in Eqs. (62) and (73) are not identical since the corresponding states $\Psi_{\Gamma, \rho}^{\min , \lambda}$ and $\Psi_{\Gamma, \rho}^{\min ^{\prime}, \mu}$ minimize different expressions. This is a direct consequence of the definitions chosen in Eqs. (52)-(55). In particular, this choice guarantees that $E_{\mathrm{Hxc}}^{e}, T_{\mathrm{MPC}}$, and $E_{\mathrm{Hc}}^{e n}$ are independent of the true external potential $U_{\text {ext }}$, i.e., these functionals are universal; all effects stemming from $U_{\text {ext }}$ are contained exclusively in the functional $U_{\mathrm{Hxc}}$.

By virtue of the above discussion, the influence of the true external potential has to be treated similar to an interaction. As already mentioned above, this complication is an immediate consequence of the necessity to transform to an internal reference system for the formulation of the MCDFT scheme. Of course, in the numerous cases discussing the properties of isolated systems, $\hat{U}$ vanishes and the MCDFT formalism reduces to the one given in [3]. If, on the other hand, a true external potential is applied to the system, approximations for $U_{\mathrm{Hxc}}$ are needed. In the simplest case, the electronic conditional density $\gamma$ is replaced by the electronic density $\rho$, leading to a Hartree-type approximation for $U_{\mathrm{Hxc}}$. Such an approximation will be especially valid in the case of well localized nuclei, as discussed later on.

\section{Analysis of the Effective Potentials}

In the last sections, the Hxc energy functional of Eq. (45) was discussed. According to Eqs. (43) and (44), this quantity gives rise to the many-body contributions of the effective MCKS potentials. Explicitly, the UHxc potentials are given by

$$
\begin{aligned}
V_{\mathrm{U}, \mathrm{Hxc}}[\Gamma, \rho](\underline{\underline{\mathbf{R}}}) & =\frac{\delta E_{\mathrm{U}, \mathrm{Hxc}}[\Gamma, \rho]}{\delta \Gamma(\underline{\underline{\mathbf{R}}})} \\
v_{\mathrm{U}, \mathrm{Hxc}}[\Gamma, \rho](\mathbf{r}) & =\frac{\delta E_{\mathrm{U}, \mathrm{Hxc}}[\Gamma, \rho]}{\delta \rho(\mathbf{r})} .
\end{aligned}
$$

Employing Eq. (58), the potentials can also be decomposed into the parts associated with the different interactions, yielding

$$
\begin{aligned}
V_{\mathrm{U}, \mathrm{Hxc}}[\Gamma, \rho](\underline{\underline{\mathbf{R}}}) & =V_{\mathrm{Hxc}}^{U}[\Gamma, \rho](\underline{\underline{\mathbf{R}}})+V_{\mathrm{Hxc}}^{e n}[\Gamma, \rho](\underline{\underline{\mathbf{R}}}) \\
& +V_{\mathrm{MPC}}[\Gamma, \rho](\underline{\underline{\mathbf{R}}}) \\
v_{\mathrm{U}, \mathrm{Hxc}}[\Gamma, \rho](\mathbf{r}) & =v_{\mathrm{Hxc}}^{U}[\Gamma, \rho](\mathbf{r})+v_{\mathrm{H}}^{e}[\rho](\mathbf{r})+v_{\mathrm{xc}}^{e}[\rho](\mathbf{r}) \\
& +v_{\mathrm{Hc}}^{e n}[\Gamma, \rho](\mathbf{r})+v_{\mathrm{MPC}}[\Gamma, \rho](\mathbf{r}),
\end{aligned}
$$

where the various potential terms on the right-hand sides of the above equations are defined in analogy to (75) and (76): The first terms on the right-hand side of Eqs. (75) and (76) represent the influence of the true external potential $\hat{U}$ and correspond to the derivatives of $U_{\mathrm{Hxc}}$ in Eq. (58). Since the electron-electron interaction is treated employing the well-known Hxc energy functional $E_{\mathrm{Hxc}}^{e}[\rho]$ from standard electronic DFT, the corresponding potentials $v_{\mathrm{H}}^{e}$ and $v_{\mathrm{xc}}^{e}$ are also identical to the familiar electronic Hartree and xc potentials. Furthermore, as for the energy functional, the potentials arising from the masspolarization and Coriolis effects are not expected to contribute significantly - at least for ground-state properties. In the following we will concentrate on the Hxc potentials arising from the electron-nuclear energy functional $E_{\mathrm{Hc}}^{e n}$.

To start with, we consider the nuclear Hc potential, defined by

$$
V_{\mathrm{Hc}}^{e n}[\Gamma, \rho](\underline{\underline{\mathbf{R}}})=\frac{\delta E_{\mathrm{Hc}}^{e n}[\Gamma, \rho]}{\delta \Gamma(\underline{\underline{\mathbf{R}}})} .
$$

Employing the representation of $E_{\mathrm{Hc}}^{e n}$ in terms of the coupling-constant averaged conditional density, Eq. 662), the nuclear potential can be split in two parts,

$$
V_{\mathrm{Hc}}^{e n}[\Gamma, \rho](\underline{\underline{\mathbf{R}}})=V_{\text {cond }}^{e n}[\Gamma, \rho](\underline{\underline{\mathbf{R}}})+V_{\mathrm{c}, \mathrm{rsp}}^{e n}[\Gamma, \rho](\underline{\underline{\mathbf{R}}})
$$

where

$$
V_{\text {cond }}^{e n}[\Gamma, \rho](\underline{\underline{\mathbf{R}}}):=\int d \mathbf{r} W_{e n}(\underline{\underline{\mathbf{R}}}, \mathbf{r}) \bar{\gamma}^{\min }[\Gamma, \rho](\mathbf{r} \underline{\underline{\mathbf{R}}})
$$

is the electrostatic potential due to the electronic conditional density and

$$
\begin{array}{r}
V_{\mathrm{c}, \mathrm{rsp}}^{e n}[\Gamma, \rho](\underline{\underline{\mathbf{R}}}):=\int d^{N_{n}} \mathbf{R}^{\prime} \Gamma\left(\underline{\underline{\mathbf{R}}}^{\prime}\right) \\
\times \int d \mathbf{r} W_{e n}\left(\underline{\underline{\mathbf{R}}}^{\prime}, \mathbf{r}\right) \frac{\delta \bar{\gamma}^{\min }[\Gamma, \rho]\left(\mathbf{r} \mid \underline{\underline{\mathbf{R}}}^{\prime}\right)}{\delta \Gamma(\underline{\underline{\mathbf{R}}})}
\end{array}
$$

defines a response-type contribution to the electronnuclear correlation potential. We note that the conditional potential $V_{\text {cond }}^{e n}$ completely determines the electronnuclear Hc energy:

$$
E_{\mathrm{Hc}}^{e n}[\Gamma, \rho]=\int d^{N_{n}} \mathbf{R} \Gamma(\underline{\underline{\mathbf{R}}}) V_{\text {cond }}^{\text {en }}[\Gamma, \rho](\underline{\underline{\mathbf{R}}}) .
$$

In the following analysis we restrict ourselves to situations where the full electron-nuclear wave function $\Psi_{\Gamma, \rho}^{\min }$ 
can be factorized into a nuclear spin function times a remainder not depending on $\underline{\underline{s}}$. This is exactly true, e.g., for diatomic molecules or when the nuclei are spin-zero bosons. In many other cases, this factorization represents a good approximation. Under these circumstances, the wave function $\Xi$ in Eq. (67) can be chosen to be independent of $\underline{\underline{s}}$, and likewise the expansion coefficients $a_{k}$ in Eq. (69), so that Eq. (71) reduces to

$$
\begin{aligned}
& E_{\mathrm{Hc}}^{e n}[\Gamma, \rho] \\
& =\int d^{N_{n}} \mathbf{R} \Gamma(\underline{\underline{\mathbf{R}}}) \sum_{k, l}\left\{\left|a_{k}[\Gamma, \rho](\underline{\underline{\mathbf{R}}})\right|^{2} \cdot \epsilon_{k}^{\mathrm{BO}}(\underline{\underline{\mathbf{R}}}) \delta_{k, l}\right. \\
& \left.+a_{k}^{\star}[\Gamma, \rho](\underline{\underline{\mathbf{R}}})\left\langle\Xi_{k}^{\mathrm{BO}}\left|\hat{T}_{n}\right| \Xi_{l}^{\mathrm{BO}}\right\rangle_{e} a_{l}[\Gamma, \rho](\underline{\underline{\mathbf{R}}})\right\}-F^{e}[\rho],
\end{aligned}
$$

Comparing Eq. (83) and (84), the conditional potential (81) can be expressed in terms of the BO-PES:

$$
\begin{aligned}
& V_{\text {cond }}^{e n}[\Gamma, \rho](\underline{\underline{\mathbf{R}}})=\sum_{k}\left|a_{k}[\Gamma, \rho](\underline{\underline{\mathbf{R}}})\right|^{2} \epsilon_{k}^{\mathrm{BO}}(\underline{\underline{\mathbf{R}}}) \\
& +\sum_{k, l} a_{k}^{\star}[\Gamma, \rho](\underline{\underline{\mathbf{R}}})\left\langle\Xi_{k}^{\mathrm{BO}}\left|\hat{T}_{n}\right| \Xi_{l}^{\mathrm{BO}}\right\rangle_{e} a_{l}[\Gamma, \rho](\underline{\underline{\mathbf{R}}}) \\
& \quad-F^{e}[\rho] .
\end{aligned}
$$

This equation provides a useful tool to interpret the effective nuclear MCKS potential: The first term in (85) is a weighted sum over different adiabatic BO-PES, whereas the second one describes adiabatic and nonadiabatic corrections to it. The last term in equation (85), $F^{e}[\rho]$, just yields a constant shift and is included in the potential to maintain the same zero-energy level within the BO and MCKS schemes. Considering the case that the BO approximation accurately describes a specific system, we realize that, in the first sum, only the lowest coefficient $a_{0}$ survives and the second sum is negligible, provided the potential is evaluated at the ground-state densities. Therefore, $V_{\mathrm{Hc}}^{e n}(\underline{\underline{\mathbf{R}}}) \approx \epsilon_{0}^{\mathrm{BO}}(\underline{\underline{\mathbf{R}}})$, and the nuclear MCKS equation reduces to the nuclear $\mathrm{BO}$ equation in the limit considered here. We emphasize, however, that the way to evaluate this potential differs in the MCKS and BO methods. Whereas, in the latter, an electronic equation has to be solved for each nuclear configuration, the MCKS potential is determined by the functional derivative $\delta E_{\mathrm{Hc}}^{e n}[\Gamma, \rho] / \delta \Gamma$. Inserting the ground-state densities then yields a potential which, as a function of $\underline{\mathbf{R}}$, is very close to the BO potential (in the case discussed here). Furthermore, we can conclude that the response part of the nuclear potential, Eq. (82), has negligible influence for such systems. If, on the other hand, nonadiabatic effects - e.g. close to level crossings - are encountered, the coefficients $a_{k}$ in Eq. (85) will, as a function of the nuclear configuration, achieve a natural diabatization. One should also note that the electronic wavefunction $\Xi$ is, in general, complex at points of degeneracy. Therefore, one obtains another contribution to the nuclear potential, which is responsible for Berry-phase effects [4] 60]. In addition, the response part of the nuclear potential might contribute appreciably. In summary, Eq. 855 shows that the (exact) nuclear effective potential reduces to the lowest-energy BO-PES, if nonadiabatic contributions can be neglected, but also contains in principle all non-BO effects. Whether or not they can be recovered in an actual application crucially depends, of course, on the level of sophistication of the approximation used for $E_{\text {Hc }}^{e n}$.

Employing again Eq. (62), the electronic potential due to the electron-nuclear interaction, defined by

$$
v_{\mathrm{Hc}}^{e n}[\Gamma, \rho](\mathbf{r}):=\frac{\delta E_{\mathrm{Hc}}^{e n}[\Gamma, \rho]}{\delta \rho(\mathbf{r})},
$$

is given by:

$$
\begin{aligned}
& v_{\mathrm{Hc}}^{e n}[\Gamma, \rho](\mathbf{r})=\int d^{N_{n}} \mathbf{R} \Gamma(\underline{\underline{\mathbf{R}}}) \\
& \times \int d \mathbf{r}^{\prime} W_{e n}\left(\underline{\underline{\mathbf{R}}}, \mathbf{r}^{\prime}\right) \frac{\delta \bar{\gamma}^{\min }[\Gamma, \rho]\left(\mathbf{r}^{\prime} \mid \underline{\underline{\mathbf{R}}}\right)}{\delta \rho(\mathbf{r})} .
\end{aligned}
$$

This expression appears rather complicated to evaluate. If, however, the nuclear probability distribution is sharply peaked around an equilibrium geometry $\underline{\underline{\mathbf{R}}}_{e q}$, only configurations around $\underline{\underline{\mathbf{R}}}_{e q}$ will substantially contribute to the above integral. Then, the calculation of the electronic Hxc potential simplifies to

$$
v_{\mathrm{Hc}}^{e n}[\Gamma, \rho](\mathbf{r}) \approx \int d^{N_{n}} \mathbf{R} \Gamma(\underline{\underline{\mathbf{R}}}) W_{e n}(\underline{\underline{\mathbf{R}}}, \mathbf{r}) .
$$

This potential represents the electrostatic (Hartree) potential due to the nuclear charge distribution acting on the electrons. Since the nuclear ground-state densities of many molecules are indeed strongly localized functions in other words: the nuclei behave almost classically - we expect the Hartree approximation for the electronic potential to be sufficiently accurate for such systems. If, on the other hand, the assumption of nicely localized nuclear densities breaks down, one needs to incorporate correlation contribution to $v_{\mathrm{Hc}}^{e n}$ arising from the electron-nuclear interaction.

\section{E. The Limit of Classical (Point-Like) Nuclei}

In this section, we investigate the limit of classical, i.e., perfectly localized nuclei. Assuming identical zero spin nuclei for the ease of notation, the nuclear density matrix reads

$$
\Gamma^{\text {class }}\left(\mathbf{R}_{1} \ldots \mathbf{R}_{N_{n}}\right)=\frac{1}{N_{n} !} \sum_{\mathcal{P}} \prod_{\alpha} \delta\left(\mathbf{R}_{\mathcal{P}(\alpha)}-\mathbf{R}_{\alpha, 0}\right)
$$

where the sum is over all $N_{n}$ ! permutations of the nuclear coordinates and $\underline{\mathbf{R}}_{0}$ denotes the positions where the nuclei are located. Note that by this classical form of the density matrix we have broken the translational and rotational symmetry of the density matrix as presented 
in Eq. (21). In the following we investigate the consequences are of this form for the diagonal of the nuclear density matrix. First, we consider the electronic density. In terms of the coupling-constant dependent conditional density, it is given by

$$
\rho(\mathbf{r})=\int d^{N_{n}} \mathbf{R} \Gamma(\underline{\underline{\mathbf{R}}}) \gamma^{\lambda}(\mathbf{r} \mid \underline{\underline{\mathbf{R}}}) .
$$

We recall that $\rho(\mathbf{r})$ does not depend on the coupling constant $\lambda$, since the external potentials are chosen such that the densities remain unchanged. Inserting Eq. (89) into (90) yields

$$
\rho(\mathbf{r})=\gamma^{\lambda}\left(\mathbf{r} \underline{\underline{\mathbf{R}}}_{0}\right),
$$

i.e., in the limit of classical nuclei, the electronic density is identical to the conditional density evaluated at the positions of the classical nuclei. This quantity, in fact, serves as the basic variable of standard electronic DFT employing the BO approximation: $\rho^{\mathrm{DFT}, \mathrm{BO}}(\mathbf{r})=\gamma\left(\mathbf{r} \mid \underline{\underline{\mathbf{R}}}_{0}\right)$. We therefore conclude that the MCDFT presented here reduces to the standard formulation of DFT in the limit of classical nuclei.

Inserting Eq. (90) into Eqs. (62) and (73), we readily obtain the expressions for $E_{\mathrm{Hc}}^{e n}$ and $U_{\mathrm{Hxc}}$ in the classical limit:

$$
\begin{aligned}
E_{\mathrm{Hc}}^{e n}\left[\Gamma^{\text {class }}, \rho\right] & =\int d \mathbf{r} \rho(\mathbf{r}) W_{e n}\left(\underline{\underline{\mathbf{R}}}_{0}, \mathbf{r}\right) \\
U_{\mathrm{Hxc}}\left[\Gamma^{\text {class }}, \rho\right] & =\int d \mathbf{r} \rho(\mathbf{r}) U_{\mathrm{ext}}\left(\underline{\underline{\mathbf{R}}}_{0}, \mathbf{r}\right) .
\end{aligned}
$$

Thus, in the limit of classical nuclei, the Hxc energy functionals reduce to the classical electrostatic (Hartree) interactions and correlation contributions vanish 32.

The corresponding electronic potentials, following from Eqs. (92) and (93) then read

$$
\begin{gathered}
v_{\mathrm{Hc}}^{e n}\left[\Gamma^{\text {class }}, \rho\right]=W_{e n}\left(\underline{\underline{\mathbf{R}}}_{0}, \mathbf{r}\right) \\
v_{\mathrm{Hxc}}^{U}\left[\Gamma^{\text {class }}, \rho\right]=U_{\text {ext }}\left(\underline{\underline{\mathbf{R}}}_{0}, \mathbf{r}\right) .
\end{gathered}
$$

The first quantity is identical to the classical Coulomb field of the nuclei, whereas the second one describes the influence of potentials applied externally to the system. Both quantites together represent the "external potential" in BO-based DFT, reflecting again its coincidence with the MCDFT in the limit of classical nuclei. From this perspective, one also might consider Eq. (88) as the natural extension of Eq. (94) to nuclear distributions which are localized but still exhibit a finite width.

\section{APPLICATIONS}

\section{A. Diatomic molecules}

Having discussed the foundation and some formal properties of the MCDFT, we proceed with the application of the theory to the case of isolated diatomic molecules.
However, as mentioned above, we treatment of the $N_{n^{-}}$ body nuclear MCKS equation has to be discussed prior to actual applications: Since "true" external potentials are absent, i.e., $\hat{U} \equiv 0$ the system is translationally invariant. Accordingly, the nuclear MCKS potential is required to behave as $V_{S, n}\left(\mathbf{R}_{1}, \mathbf{R}_{2}\right)=V_{S, n}\left(\mathbf{R}_{1}-\mathbf{R}_{2}\right)$. The nuclear equation has then the form

$$
\begin{aligned}
(- & \frac{1}{2 M_{1}} \nabla_{\mathbf{R}_{1}}^{2}-\frac{1}{2 M_{2}} \nabla_{\mathbf{R}_{2}}^{2} \\
& \left.+V_{S, n}\left(\mathbf{R}_{1}-\mathbf{R}_{2}\right)-\epsilon_{n}\right) \chi\left(\mathbf{R}_{1} s_{1}, \mathbf{R}_{2} s_{2}\right)=0
\end{aligned}
$$

Then the nuclear CM motion can be separated off and the problem can be reformulated in terms of the internuclear separation $\mathbf{R}:=\mathbf{R}_{2}-\mathbf{R}_{1}$. The nuclear function $\chi$ has the general form

$$
\chi\left(\mathbf{R}_{1} s_{1}, \mathbf{R}_{2} s_{2}\right)=\eta\left(\mathbf{R}_{C M}\right) \xi\left(\mathbf{R}_{1}-\mathbf{R}_{2}\right) \theta\left(s_{1}, s_{2}\right)
$$

where $\eta\left(\mathbf{R}_{C M}\right)$ is a plane wave state depending on the center-of-mass coordinate $\mathbf{R}_{C M}=\left(M_{1} \mathbf{R}_{1}+\right.$ $\left.M_{2} \mathbf{R}_{2}\right) /\left(M_{1}+M_{2}\right)$ and is explicitly given by

$$
\eta\left(\mathbf{R}_{C M}\right)=\frac{1}{\sqrt{V}} e^{i \mathbf{k} \cdot \mathbf{R}_{C M}}
$$

where $V$ is the total volume of the system. The relative wavefunction $\xi\left(\mathbf{R}_{1}-\mathbf{R}_{2}\right)$ satisfies the nuclear MCKS equation

$$
\left(-\frac{\nabla_{\mathbf{R}}^{2}}{2 \mu_{n}}+V_{S, n}(\mathbf{R})-\epsilon_{n}\right) \xi(\mathbf{R})=0,
$$

where $\mu_{n}=M_{1} M_{2} /\left(M_{1}+M_{2}\right)$ denotes the reduced nuclear mass. The function $\theta$ is a nuclear spin function depending on the type of nuclear species. For instance, for the $H_{2}$ with two protons as nuclei the nuclear ground state is that of para-hydrogen where the function $\theta$ is an antisymmetric spin function and consequently the function $\xi$ must be even, i.e. $\xi(\mathbf{R})=\xi(-\mathbf{R})$, to preserve overall anti-symmetry of the wavefunction under the interchange of the two protons. For the density matrix $\Gamma$, one obtains after integrating out the spin function

$$
\Gamma\left(\mathbf{R}_{1}, \mathbf{R}_{2}\right)=\frac{1}{V}\left|\xi\left(\mathbf{R}_{1}-\mathbf{R}_{2}\right)\right|^{2} \equiv \frac{1}{V} \Gamma(\mathbf{R}) .
$$

Therefore, the diagonal of the nuclear density matrix, which we often - and somewhat unprecisely - refer to as "nuclear density" is indeed a single-particle quantity describing the probability of finding the two nuclei separated by $\mathbf{R}$.

It remains to discuss the electronic coordinates. For our diatomic molecule we determine the Euler angles by the requirement that the internuclear axis be parallel to the $z$-axis in the body-fixed frame, i.e. $\mathcal{R}\left(\mathbf{R}_{1}-\mathbf{R}_{2}\right)=R \mathbf{e}_{z}$ , where $R=\left|\mathbf{R}_{1}-\mathbf{R}_{2}\right|$. For the special case of the diatomic molecule only two Euler angles are needed to specify the rotation matrix $\mathcal{R}$. The electronic coordinates in the body-fixed frame are then obtained using Eq.(9). 
With this transformation the electron-nuclear attraction attains the form

$$
W_{e n}(\mathbf{R}, \mathbf{r})=-\frac{Z_{1}}{\left|\mathbf{r}-\frac{M_{2}}{M_{\mathrm{nuc}}} R \mathbf{e}_{z}\right|}-\frac{Z_{2}}{\left|\mathbf{r}+\frac{M_{1}}{M_{\mathrm{nuc}}} R \mathbf{e}_{z}\right|} .
$$

As for any other DFT, explicit approximations have to be employed for the energy functional $E_{\mathrm{U}, \mathrm{Hxc}}[\Gamma, \rho]$ of Eq.(58). Since no "true" external potentials are present in the case discussed here, $U_{\mathrm{Hxc}}[\Gamma, \rho]$ vanishes identically. Furthermore, following Sec. IIIA the purely electronic part of the energy functional can be treated by using the familiar approximations for the electronic xc energy functional $E_{\mathrm{xc}}^{e}[\rho]$. For all systems containing more than one electron, we will employ the well-known LDA approximation. We emphasize that it is not the purpose of this work to investigate new approximations for the electronic xc energy functional. Instead, we aim at an analysis of the previously not much studied Hc energy functional arising from the electron-nuclear interaction. To that end, we restrict ourselves to work within the LDA approximation for $E_{\mathrm{xc}}^{e}[\rho]$; different - and more sophisticated - approximations for the electronic xc energy functional would only result in some minor quantitative changes of the analysis presented below. Furthermore, we note that the mass-polarization and Coriolis effects are not expected to contribute substantially to groundstate properties. Therefore, only the diagonal part of the mass-polarization term which leads to a reduced mass in the electronic MCKS equation is accounted for and the remaining parts are neglected in all practical calculations. With these assumptions the electronic Kohn-Sham equations for our problem then attain the form

$$
\left(-\frac{\nabla^{2}}{2 \mu_{e}}+v_{S, e}[\Gamma, \rho](\mathbf{r})-\epsilon_{e, j}\right) \varphi_{j}(\mathbf{r})=0
$$

where

$$
v_{S, e}[\Gamma, \rho](\mathbf{r})=v_{\mathrm{Hc}}^{e n}[\Gamma, \rho](\mathbf{r})+v_{\mathrm{H}}^{e}[\rho](\mathbf{r})+v_{\mathrm{xc}}^{e}[\rho](\mathbf{r})
$$

and $\mu_{e}=\left(M_{1}+M_{2}\right) /\left(M_{1}+M_{2}+1\right)$. The approximations used for $v_{\mathrm{Hc}}^{e n}$ will be discussed in the subsequent sections. Within the same assumptions the effective potential in the nuclear equations will be of the form

$$
V_{S, n}[\Gamma, \rho](\mathbf{R})=W_{e e}(\mathbf{R})+V_{\mathrm{Hc}}^{e n}[\Gamma, \rho](\mathbf{R})
$$

where $W_{e e}(\mathbf{R})=Z_{1} Z_{2} / R$.

We finally note that the diatomic molecule is a particularly convenient case for studying the nuclear effective potential $V_{S, n}$ since for this case for a given density $\Gamma(\mathbf{R})$ the potential $V_{S, n}$ is easily constructed from inversion of Eq.(99):

$$
V_{S, n}(\mathbf{R})=\epsilon_{n}+\frac{1}{2 \mu_{n}} \frac{\nabla_{\mathbf{R}}^{2} \sqrt{\Gamma(\mathbf{R})}}{\sqrt{\Gamma(\mathbf{R})}}
$$

We have done this for a one-dimensional model of the $\mathrm{H}_{2}^{+}$ for which the exact nuclear density $\Gamma$ can be obtained by numerical integration of the full many-body Schrödinger equation. In this way the exact nuclear potential $V_{S, n}$ for this problem was obtained 13. For this case we found the exact $V_{S, n}$ to be almost identical to the BO potential except for the case when the nuclear masses were taken to be artificially small. This illustrates the point discussed before: in situations where the $\mathrm{BO}$ approximation works well, the nuclear potential $V_{S, n}$ will be close to the $\mathrm{BO}$ potential. For this reason the $\mathrm{BO}$ potentials of the $\mathrm{H}_{2}$ and $\mathrm{H}_{2}^{+}$molecules that we will study below will be a good reference to test our approximations for the electron-nuclear correlation functional. Of course, having obtained a good approximate functional its main field of applicability will be cases where the BO approximation does not work well.

To conclude these introductory remarks, the numerical implementation of the MCKS equations (99) and (102) is briefly described. Since Coriolis effects are neglected, the effective nuclear potential is spherically symmetric and the angular part can be treated analytically. The remaining radial nuclear MCKS equation is numerically solved on a one-dimensional grid. Furthermore, we observe that the $z$-component of the electronic angular momentum is a conserved quantity. Hence, the electronic MCKS equation is rewritten in terms of cylindrical coordinates. For axial-symmetric electronic potentials, the angular part can be integrated out and we are left with a two-dimensional problem. The resulting Hamiltonian is then discretized on a uniform rectangular grid and numerically diagonalized by employing the Lanczos algorithm [48]. Due to the use of finite uniform grids, the regions around the nuclei are not sampled with high accuracy, leading to typical discretization errors of about $0.1 \%$ for the systems discussed later in this section. Both the nuclear and the electronic equation are solved simultaneously until self-consistency is achieved.

\section{B. The Hartree Approximation for the electron-nuclear energy functional}

It remains to find explicit approximations for the electron-nuclear $\mathrm{Hc}$ energy functional $E_{\mathrm{Hc}}^{e n}[\Gamma, \rho]$. In the simplest case, the electron-nuclear interaction is approximated by the Hartree energy functional, defined by

$$
\begin{aligned}
E_{\mathrm{H}}^{e n}[\Gamma, \rho] & :=\int d \mathbf{R}_{1} d \mathbf{R}_{2} d \mathbf{r} \Gamma(\underline{\underline{\mathbf{R}}}) W_{e n}(\underline{\underline{\mathbf{R}}}, \mathbf{r}) \rho(\mathbf{r}) \\
& =\int d \mathbf{R} d \mathbf{r} \Gamma(\mathbf{R}) W_{e n}(\mathbf{R}, \mathbf{r}) \rho(\mathbf{r}) .
\end{aligned}
$$

where in the second step we changed to relative $\mathbf{R}$ and center-of-mass $\mathbf{R}_{C M}$ coordinates and performed the integration over $\mathbf{R}_{C M}$ which eliminated the inverse volume prefactor in Eq.(100). By virtue of Eq. (106), the Hc energy functional $E_{\mathrm{Hc}}^{e n}[\Gamma, \rho]$ is thus replaced by the classical electrostatic interactions of the corresponding charge distributions and correlation contributions are neglected. 


\begin{tabular}{|c|c|c|c|c|}
\hline & $\mathrm{BO}$ & Hartree & $\mathrm{OAO}$ & $\mathrm{SAO}$ \\
\hline$-E_{0}$ & 1.130 & 1.121 & 1.122 & 1.124 \\
\hline$T_{S}$ & & 1.069 & 1.063 & 1.049 \\
\hline$E_{\mathrm{Hxc}, e}^{e}$ & & 0.627 & 0.625 & 0.623 \\
\hline$-E_{\mathrm{Hc}}^{e n}$ & & 3.496 & 3.487 & 3.471 \\
\hline$W_{n n}$ & & 0.679 & 0.676 & 0.676 \\
\hline$\langle R\rangle$ & 1.49 & 1.48 & 1.49 & 1.50 \\
\hline$\left\langle R^{2}\right\rangle$ & 2.25 & & 2.24 & 2.28 \\
\hline$\omega\left[\mathrm{cm}^{-1}\right]$ & 4137 & 7945 & 7047 & 4282 \\
\hline
\end{tabular}

TABLE I: Summary of results for the $\mathrm{H}_{2}$ molecule obtained from self-consistent solutions of the MCKS scheme employing various approximations. For comparison, results from $B O$ calculations are added. The electronic interaction is treated within the xcLDA. All numbers (except $\omega$ ) in atomic units.

Evidently Eq. (106) can also be derived from a product (mean-field) ansatz for the electron-nuclear part of the total wavefunction. In fact, such a mean-field description of the electron-nuclear interaction has been proposed in [49, 50] to study the protonic structure of molecules.

From the Hartree-energy functional (106), the corresponding potentials, defined in (79) and (86), are readily calculated:

$$
\begin{aligned}
V_{\mathrm{H}}^{e n}(\mathbf{R}) & =\int d \mathbf{r} W_{e n}(\mathbf{R}, \mathbf{r}) \rho(\mathbf{r}) \\
v_{\mathrm{H}}^{e n}(\mathbf{r}) & =\int d \mathbf{R} W_{e n}(\mathbf{R}, \mathbf{r}) \Gamma(\mathbf{R}) .
\end{aligned}
$$

We note that, within the Hartree approximation, the nuclear response potential (82) vanishes and the conditional potential (81) is given by (107). With these Hartree potentials inserted in expressions 103 and (104), the MCKS equations (99) and (102) are solved self-consistently as described above.

\section{Results}

In the following, the application of the Hartree approximation to the $\mathrm{H}_{2}$ and $\mathrm{H}_{2}^{+}$molecules is discussed.

In tables $\square$ and $\Pi$ a selection of results is presented. Since the BO approach provides an excellent approximation for the system under consideration, we also added, for comparison, the results obtained from the $\mathrm{BO}$ calculation (employing the very same numerical procedure discribed above). The ground-state results are found to be surprisingly good for both molecules. Compared to the $\mathrm{BO}$ results listed in the first column of the tables, the total ground-state energy and the geometry, represented by the mean internuclear distance $\langle R\rangle$, are reproduced up to within an accuracy of about $1 \%$ by the Hartree method. However, turning towards the harmonic con-

\begin{tabular}{|c|c|c|c|c|}
\hline & $\mathrm{BO}$ & Hartree & $\mathrm{OAO}$ & $\mathrm{SAO}$ \\
\hline$-E_{0}$ & 0.598 & 0.591 & 0.595 & 0.581 \\
\hline$T_{S}$ & & 0.591 & 0.583 & 0.574 \\
\hline$-E_{\mathrm{Hc}}^{e n}$ & & 1.673 & 1.662 & 1.642 \\
\hline$W_{n n}$ & & 0.491 & 0.485 & 0.487 \\
\hline$\langle R\rangle$ & 2.07 & 2.05 & 2.08 & 2.08 \\
\hline$\left\langle R^{2}\right\rangle$ & 4.30 & 4.22 & 4.37 & 4.39 \\
\hline$\omega\left[\mathrm{cm}^{-1}\right]$ & 2297 & 5191 & 3248 & 2232 \\
\hline
\end{tabular}
stants, we realize that the Hartree result is off by about
TABLE II: Summary of results for the $\mathrm{H}_{2}^{+}$molecule obtained from self-consistent solutions of the MCKS scheme employing various approximations. For comparison, results from $B O$ calculations are added. All numbers (except $\omega$ ) in atomic units.

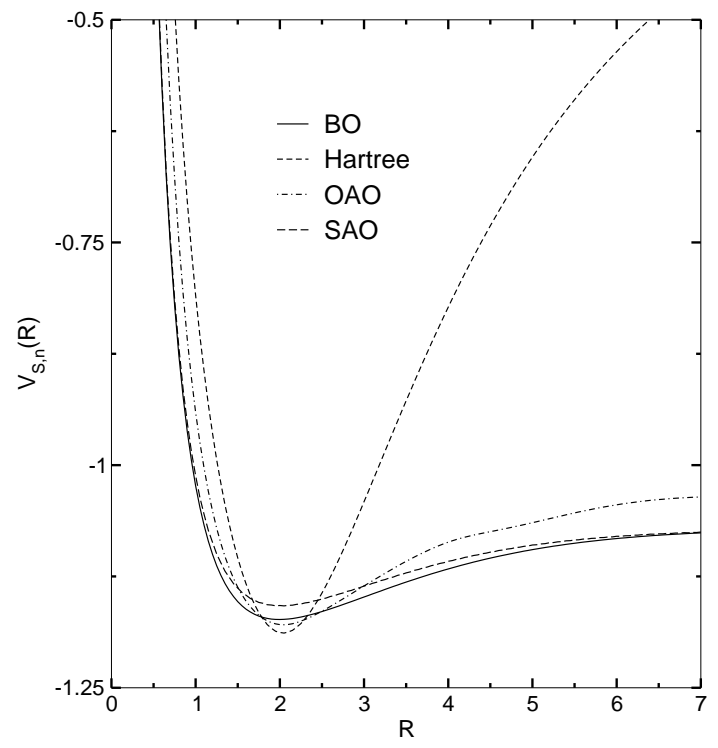

FIG. 1: Effective nuclear potential $V_{S, n}(R)$ for the $\mathrm{H}_{2}^{+}$molecule obtained from self-consistent solutions of the $M C K S$ scheme employing various approximations. For comparison, results from exact and $B O$ calculations are added. In atomic units.

a factor of two for both $H_{2}$ and $H_{2}^{+}$. Since $\omega$ measures the curvature of the effective nuclear potentials at the minimum, we may expect larger deviations in this quantity. In fact, this is confirmed by Fig. 3 and Fig. 1 where the effective nuclear MCKS potential is plotted for the $\mathrm{H}_{2}$ and $\mathrm{H}_{2}^{+}$molecule. Clearly, the Hartree potential is satisfactorily only in a small region around the minimum of the potential - which, however, is sufficient for good results for the total ground-state energy or geometry. For larger $R$, the potential grows much to fast such that the depth of the potentials is largly overestimated.

In fact from the large $R$ behavior of Eq.(107) we see that the asymptotic behavior of the effective potential in 


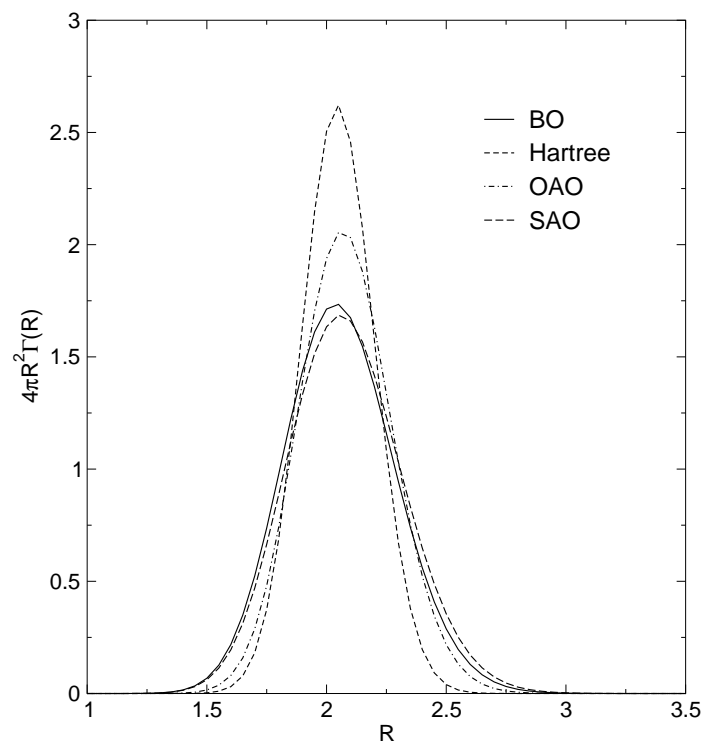

FIG. 2: Radial nuclear density $4 \pi R^{2} \Gamma(R)$ for the $H_{2}^{+}$molecule obtained from self-consistent solutions of the $M C K S$ scheme employing various approximations. For comparison, results from exact and BO calculations are added. In atomic units.

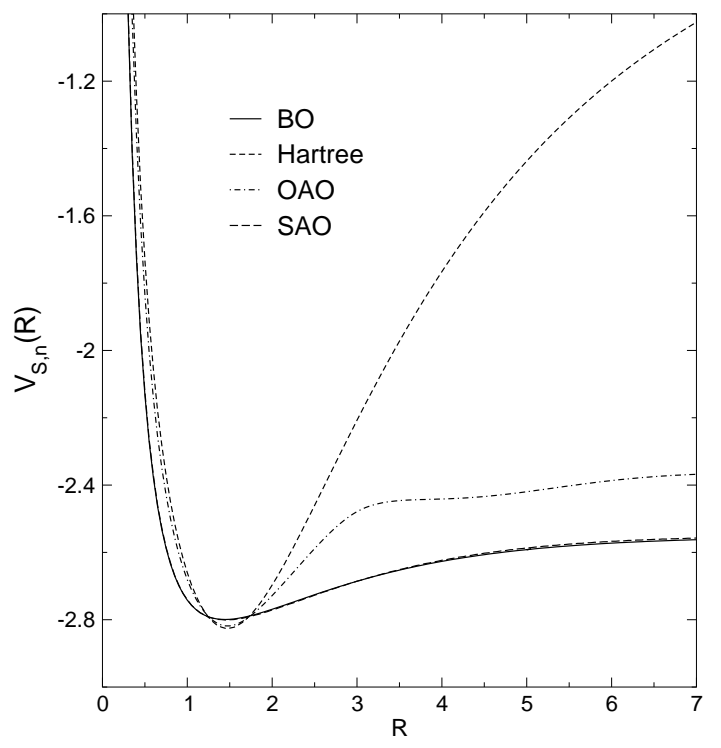

FIG. 3: Effective nuclear potential $V_{S, n}(R)$ for the $\mathrm{H}_{2}$ molecule obtained from self-consistent solutions of the MCKS scheme employing various approximations. For comparison, results from a $B O$ calculations is added. In atomic units.

the Hartree approximation is given by

$$
V_{S, n}(\mathbf{R}) \stackrel{R \rightarrow \infty}{\longrightarrow}\left(Z_{1} Z_{2}-N_{e} M_{\mathrm{nuc}}\left(\frac{Z_{1}}{M_{2}}+\frac{Z_{2}}{M_{1}}\right)\right) \frac{1}{R}
$$

whereas the $\mathrm{BO}$ potential approaches a finite value in this limit. As a consequence of the steep rise of the Hartree potential, the corresponding nuclear densities of both molecules, shown in Fig 2 and Fig 4 are much more localized than the $\mathrm{BO}$ ones and reflect the wrong shape

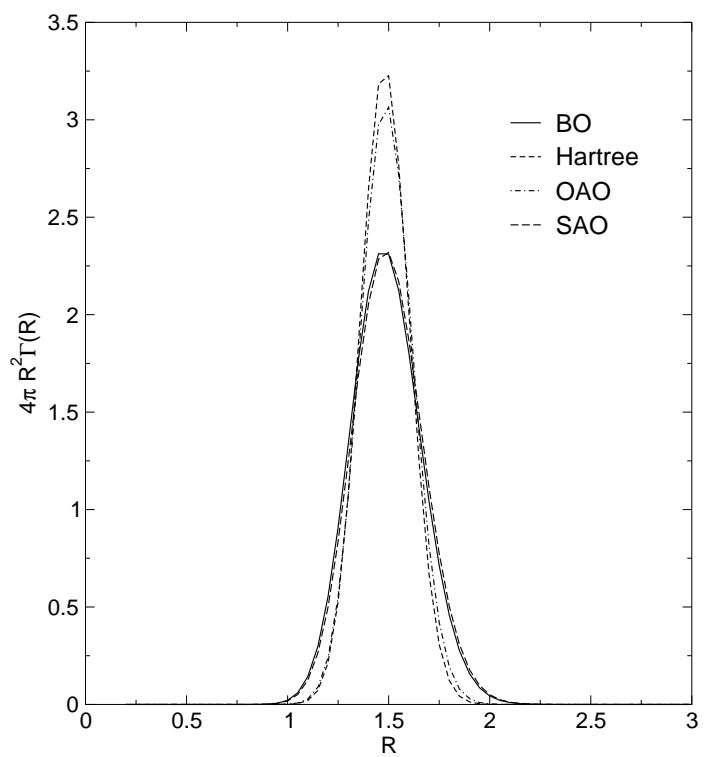

FIG. 4: Radial nuclear density $4 \pi R^{2} \Gamma(R)$ for the $H_{2}$ molecule obtained from self-consistent solutions of the MCKS scheme employing various approximations. For comparison, results from exact and BO calculations are added. In atomic units.

of the effective nuclear potential.

To understand the origin of these deviations in more detail, we reconsider the expression for the Hxc energy functional, Eq. (62). A comparison of this equation with the Hartree energy functional (106) shows that the Hartree approximation for the electronic conditional density is

$$
\gamma_{\mathrm{H}}(\mathbf{r} \mid \mathbf{R})=\rho(\mathbf{r}) \quad \forall \mathbf{R},
$$

i.e., the conditional density is independent of $\mathbf{R}$ in the Hartree approach. In the case that the nuclear density is a well-localized function, the approximation (110) is justified for $\mathbf{R} \approx \mathbf{R}_{e q}$, (where $\mathbf{R}_{e q}$ denotes the equilibrium separation) since many quantities of interest then only depend on internuclear separations close to $\mathbf{R}_{e q}$. Thus the approximation (110) leads to reasonable results, as reported above. However, Eq. (110) fails for large $\left|\mathbf{R}-\mathbf{R}_{e q}\right|$. This is illustrated in Fig. [5 where we sketched a typical behavior of the electronic density $\rho(\mathbf{r})$ and the conditional density $\gamma(\mathbf{r} \mid \mathbf{R})$ for $|\mathbf{R}| \approx 6$ a.u. As a consequence, the Hartree approximation cannot be expected to be accurate for $R>\left|\mathbf{R}_{e q}\right|$, explaining the deviations discussed in Sec. IVB

To summarize, the Hartree approximation provides a fair estimate for ground-state properties of diatomic molecules, such as the total ground-state energy or the equilibrium geometry. However, the nuclear Hartree potential only reproduces the position of the minimum but fails to correctly describe the shape of the exact MCKS potential. If one is interested in quantities depending more sensibly on the shape of the nuclear potential, the Hartree method thus needs to be improved. 


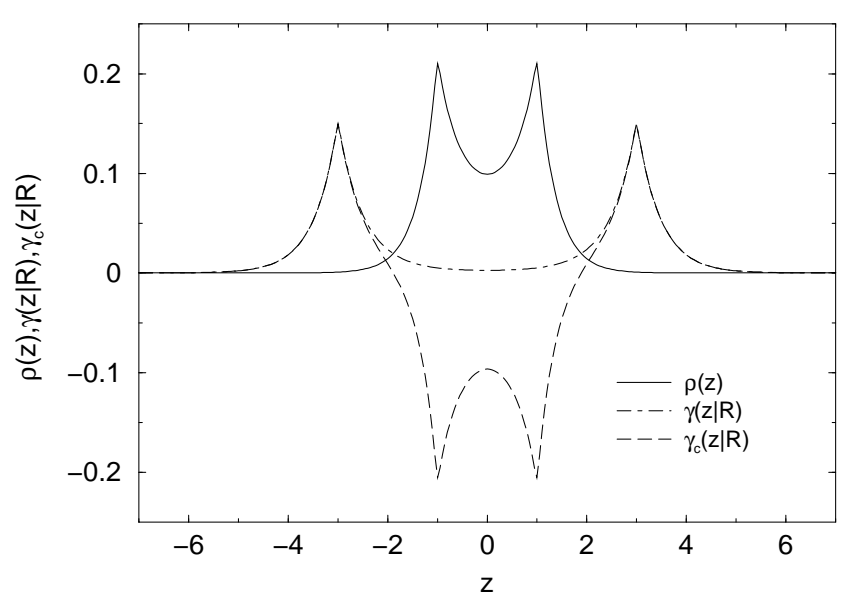

FIG. 5: Typical behavior of the electronic density $\rho(z)$ and the conditional density $\gamma(z \mid R) \approx 6$ a.u.) for a diatomic molecule, plotted along the internuclear $(z)$ axis. In atomic units.

\section{An Approach Based on Atomic Orbitals}

From the analysis of the preceding sections, we were lead to the conclusion that one needs to go beyond the Hartree approximation for the electron-nuclear energy functional $E_{\mathrm{Hxc}}^{e n}[\Gamma, \rho]$. Moreover, in view of the fact that the deviations between the Hartree and the exact (BO) potentials are rather large, the Hartree potential is not necessarily a good starting point for approximations - in contrast to the situation for the electron-electron interaction. This is particularily true for properties which do not only depend on internuclear distances close to $R_{e q}$. For instance, to go beyond the Hartree approximation, it might be tempting (as usually done for electronic correlations in standard DFT) to define a "hole density"

$$
\gamma_{c}(\mathbf{r} \mid \mathbf{R}):=\gamma(\mathbf{r} \mid \mathbf{R})-\rho(\mathbf{r})
$$

which measures the deviations of the density from the conditional density. Inserting this definition into Eq. (62) then leads to partitioning of the Hartree-correlation functional into a Hartree and a correlation part. However, as displayed in Fig.(5) the hole function $\gamma_{c}(\mathbf{r} \mid \mathbf{R})$ is large for almost all $\mathbf{R}$ (except $\mathbf{R} \approx \mathbf{R}_{\text {eq }}$ ) and has to account for large correlation corrections. Therefore at the present point, it appears most promising to approximate the conditional density directly. To that end, we first recall that $\gamma(\mathbf{r} \mid \mathbf{R})$ represents the probability of finding an electron at $\mathbf{r}$, provided the nuclei are separated by $\mathbf{R}$. If we would go back to a $\mathrm{BO}$ description such a quantity would be naturally described by the electronic density calculated within the BO approach as described in Eq.(91) in which case we have

$$
\gamma^{B O}(\mathbf{r} \mid \mathbf{R})=\sum_{j=1}^{N_{e}}\left|\xi_{\mathbf{R}, j}^{B O}(\mathbf{r})\right|^{2}
$$

where $\xi_{\mathbf{R}, j}^{B O}$ are the electronic single-particle orbitals for internuclear distance $\mathbf{R}$ as in standard density functional theory. In order to obtain Eq. (112) in the BO-limit we approximate the conditional density $\gamma$ in the spirit of a linear combination of atomic orbitals (LCAO) approach, i.e.,

$$
\gamma(\mathbf{r} \mid \mathbf{R}) \approx \sum_{j=1}^{N_{e}} \gamma_{j}(\mathbf{r} \mid \mathbf{R})
$$

where

$$
\gamma_{j}(\mathbf{r} \mid \mathbf{R})=\frac{1}{2 \nu_{j}(\mathbf{R})}\left|\phi_{j}^{A}\left(\mathbf{r}_{A}\right)+\phi_{j}^{B}\left(\mathbf{r}_{B}\right)\right|^{2},
$$

where $\phi_{j}^{A / B}(\mathbf{r})$ denotes an atomic-type orbital and the factor $\nu_{j}(\mathbf{R})$ is included to ensure normalization of the orbital conditional density, i.e.

$$
1=\int d \mathbf{r} \gamma_{j}(\mathbf{r} \mid \mathbf{R})
$$

This ensures normalization of the electronic Kohn-Sham orbitals in the $\mathrm{BO}$ limit. Instead of following the standard LCAO approach where $\gamma(\mathbf{r} \mid \mathbf{R})$ is constructed from given atomic orbitals $\left\{\phi_{j}^{A / B}\right\}$, we will determine suitably "optimized" atomic orbitals (OAO) from given densities $\Gamma$ and $\rho$, i.e., the atomic orbitals are represented as functionals of the densities: $\phi_{j}^{A / B}(\mathbf{r})=\phi_{j}^{A / B}[\Gamma, \rho](\mathbf{r})$. Inserting these orbitals in (114) then leads to an approximation of $\gamma[\Gamma, \rho]$ and therefore, by virtue of Eq. [62), to an approximation of $E_{\mathrm{Hc}}^{e n}[\Gamma, \rho]$ as functionals of the densities $\Gamma$ and $\rho$.

In order to find the $\mathrm{OAO}$, we first note that, given atomic orbitals $\phi_{j}^{A}(\mathbf{r})$ and $\phi_{j}^{B}(\mathbf{r})$, normalized bonding and antibonding molecular orbitals for a fixed internuclear distance $R$ can be obtained from

$$
\varphi_{\mathbf{R}, j}^{ \pm}(\mathbf{r})=\frac{1}{\sqrt{2\left(1 \pm S_{j}(\mathbf{R})\right)}}\left(\phi_{j}^{A}\left(\mathbf{r}_{A}\right) \pm \phi_{j}^{B}\left(\mathbf{r}_{B}\right)\right),
$$

where $S_{j}(\mathbf{R}):=\int d \mathbf{r} \phi_{j}^{A}\left(\mathbf{r}_{A}\right) \phi_{j}^{B}\left(\mathbf{r}_{B}\right)$ denotes the overlap integral and the atomic orbitals are assumed to be real. For the purpose of the section, we are concerned with the reverse problem: Given molecular MCKS orbitals, how can we construct corresponding atomic orbitals?

As the crucial idea, we identify the electronic MCKS orbitals $\left\{\varphi_{j}(\mathbf{r})\right\}$, i.e., the solutions of the electronic MCKS equation (102), with the bonding and antibonding orbitals of Eq. (116) evaluated at the mean internuclear distance $\langle R\rangle$ :

$$
\begin{aligned}
\varphi_{j}(\mathbf{r}) & \equiv \varphi_{\langle R\rangle, j}^{+}(\mathbf{r}) \\
\varphi_{\bar{j}}(\mathbf{r}) & \equiv \varphi_{\langle R\rangle, j}^{-}(\mathbf{r}),
\end{aligned}
$$

where $\varphi_{\bar{j}}$ denotes the antibonding counterpart of $\varphi_{j}$. Using Eqs. (116) and (117), we can solve for the atomic orbitals, yielding

$$
\phi_{j}^{A}\left[\Gamma,\left\{\varphi_{j}\right\}\right](\mathbf{r})=\sqrt{\frac{1+S_{j}}{2}} \varphi_{j}\left(\mathbf{r}+\frac{M_{2}}{M_{\mathrm{nuc}}}\langle R\rangle \mathbf{e}_{z}\right)
$$




$$
\begin{aligned}
& \left.+\sqrt{\frac{1-S_{j}}{2}} \varphi_{\bar{j}}\left(\mathbf{r}+\frac{M_{2}}{M_{\mathrm{nuc}}}\langle R\rangle \mathbf{e}_{z}\right\rangle 119\right) \\
\phi_{j}^{B}\left[\Gamma,\left\{\varphi_{j}\right\}\right](\mathbf{r}) & =\sqrt{\frac{1+S_{j}}{2}} \varphi_{j}\left(\mathbf{r}-\frac{M_{1}}{M_{\mathrm{nuc}}}\langle R\rangle \mathbf{e}_{z}\right) \\
& \left.-\sqrt{\frac{1-S_{j}}{2}} \varphi_{\bar{j}}\left(\mathbf{r}-\frac{M_{1}}{M_{\mathrm{nuc}}}\langle R\rangle \mathbf{e}_{z}\right\rangle 1.20\right)
\end{aligned}
$$

The value of the overlap $S_{j}=S_{j}(\langle R\rangle)$ is not determined by the above procedure and has to be supplied additionally. A simple estimate is obtained from the overlap of unperturbed (hydrogenic) orbitals. Using this prescription, the atomic orbitals can be calculated from Eqs. (119) and (120). We therefore determined the atomic orbitals as functionals of the nuclear density and the electronic MCKS orbitals or, by virtue of the MCHK theorem, as implicit functionals of the densities $\Gamma$ and $\rho$.

For the case of homonuclear diatomic molecules, the above equations yield $\phi_{j}^{A}(\mathbf{r})=\phi_{j}^{B}(-\mathbf{r})$. We note that the atomic orbitals are not required to have a definite symmetry with respect to parity transformations. As a matter of fact, we do not expect them to be symmetric; instead, one may view them as orbitals which are centered on one nucleus and polarized by the presence of the second nucleus. Of course, for homonuclear molecules, the linear combinations (116) are properly symmetrized, i.e., the molecular orbitals can be classified either as gerade or as ungerade states.

Employing Eqs. (119) and (120), we readily obtain an approximation of the conditional density as an (implicit) functional of the densities is obtained:

$$
\begin{aligned}
& \gamma^{\mathrm{OAO}}\left[\Gamma,\left\{\varphi_{j}\right\}\right](\mathbf{r} \mid \mathbf{R}) \\
& =\sum_{j=1}^{N_{e}} \frac{1}{2 \nu_{j}(\mathbf{R})}\left|\phi_{j}^{A}\left[\Gamma,\left\{\varphi_{j}\right\}\right]\left(\mathbf{r}_{A}\right)+\phi_{j}^{B}\left[\Gamma,\left\{\varphi_{j}\right\}\right]\left(\mathbf{r}_{B}\right)\right|^{2} .
\end{aligned}
$$

In the asymptotic $R \rightarrow \infty$ regime, Eq. (122) reduces to the correct asymptotic form, i.e., the conditional density is given by the sum of two atomic densities However, for the self-consistent ground-state solution of the MCKS scheme employing Eq. (122), the atomic orbitals are not the unperturbed orbitals representing the ground state of the dissociated fragments, but rather polarized orbitals which are optimized for the molecular ground state. Therefore, even for $R \rightarrow \infty$, the conditional density $\gamma^{\mathrm{OAO}}$ is not exact, although it should improve on the Hartree behavior.

Employing Eq. (122), we obtain an expression for the electron-nuclear interaction energy.

$$
\begin{aligned}
& W_{e n}^{\mathrm{OAO}}[\Gamma, \rho]=\int d \mathbf{R} \Gamma(\mathbf{R}) \\
& \times \quad \int d \mathbf{r} W_{e n}(\mathbf{R}, \mathbf{r}) \gamma^{\mathrm{OAO}}\left[\Gamma,\left\{\varphi_{j}[\rho]\right\}\right](\mathbf{r} \mid \mathbf{R}) .
\end{aligned}
$$

In order to calculate the Hc energy functional from this expression we could perform the coupling-constant integration by using an approximation for the $\lambda$-dependence of the conditional density in Eq.(61). Alternatively, we employ Eq. (59), yielding

$$
\begin{aligned}
& E_{\mathrm{Hc}}^{\mathrm{OAO}}[\Gamma, \rho]=\int d \mathbf{R} \Gamma(\mathbf{R}) \\
& \times\left(\int d \mathbf{r} W_{e n}(\mathbf{R}, \mathbf{r}) \gamma^{\mathrm{OAO}}(\mathbf{r} \mid \mathbf{R})+F^{e}\left[\gamma^{\mathrm{OAO}}\right](\mathbf{R})-F^{e}[\rho]\right) .
\end{aligned}
$$

where nonadiabatic terms have been neglected and $F^{e}$ is the universal electronic functional defined in (52). Eq. (124) represents the central result of this section. In order to use this approximation in a self-consistent MCKS calculation, the effective potentials have to be calculated. The nuclear conditional potential is readily evaluated, yielding

$$
\begin{aligned}
V_{\text {cond }}^{\mathrm{OAO}}(\mathbf{R}) & =V_{\text {cond, } \mathrm{W}}^{\mathrm{OAO}}(\mathbf{R})+V_{\text {cond, } \mathrm{T}}^{\mathrm{OAO}}(\mathbf{R}) \\
& +V_{\text {cond }, \mathrm{H}}^{\mathrm{OAO}}(\mathbf{R})+V_{\text {cond }, \mathrm{xc}}^{\mathrm{OAO}}(\mathbf{R})
\end{aligned}
$$

where

$$
\begin{aligned}
V_{\text {cond }, \mathrm{W}}^{\mathrm{OAO}}(\mathbf{R}) & =\int d \mathbf{r} W_{e n}(\mathbf{R}, \mathbf{r}) \gamma^{\mathrm{OAO}}(\mathbf{r} \mid \mathbf{R}) \\
V_{\mathrm{cond}, \mathrm{T}}^{\mathrm{OAO}}(\mathbf{R}) & =\frac{1}{8} \sum_{j} \int d \mathbf{r} \frac{\left|\nabla \gamma_{j}^{\mathrm{OAO}}(\mathbf{r} \mid \mathbf{R})\right|^{2}}{\gamma_{j}^{\mathrm{OAO}}(\mathbf{r} \mid \mathbf{R})} \\
& -T_{S, e}[\rho] \\
V_{\mathrm{cond}, \mathrm{H}}^{\mathrm{OAO}}(\mathbf{R}) & =\frac{1}{2} \iint d \mathbf{r} d \mathbf{r}^{\prime} \frac{\gamma^{\mathrm{OAO}}(\mathbf{r} \mid \mathbf{R}) \gamma^{\mathrm{OAO}}\left(\mathbf{r}^{\prime} \mid \mathbf{R}\right)}{\left|\mathbf{r}-\mathbf{r}^{\prime}\right|} \\
& -E_{\mathrm{H}}^{e}[\rho] \\
V_{\text {cond,xc }}^{\mathrm{OAO}}(\mathbf{R}) & =E_{\mathrm{xc}}^{e}\left[\gamma^{\mathrm{OAO}}\right](\mathbf{R})-E_{\mathrm{xc}}^{e}[\rho] .
\end{aligned}
$$

The first term on the right-hand side of Eq. (125) represents the functional derivative of $W_{e n}^{\mathrm{OAO}}[\Gamma, \rho]$ with respect to the nuclear density. The remaining terms are responsible for the $R$-dependence of the electronic contributions to the Hxc energy functional. The functional derivative of Eq. (124) is defined up to an arbitrary constant. For this reason the terms (127)-(129) are defined such that they vanish when $\gamma(\mathbf{r} \mid \mathbf{R}) \approx \rho(\mathbf{r})$. The nuclear response potential is neglected for the reasons explained already above. In order to calculate the electronic potential from (124), one would have to resort to the optimized effective potential (OEP) method [51, 52, 53], since the $\mathrm{OAO}$ energy functional depends explicitly on the electronic MCKS orbitals $\left\{\varphi_{j}\right\}$ and therefore implicitly on the electronic density. This, however, would lead to a rather complicated integral equation. On the other hand, it was shown in Sec. IVB that the electronic Hartree potential is sufficiently accurate for the systems considered here. Therefore, $v_{\mathrm{H}}^{e n}(\mathbf{r})$ will be used as an approximation for the electronic Hc potential in the current context, too. Having derived the effective potentials, the MCKS scheme is solved self-consistently.

\section{Results}

In the following, the results of the OAO appoach are presented for the $H_{2}^{+}$and the $H_{2}$ molecules. First of all, 


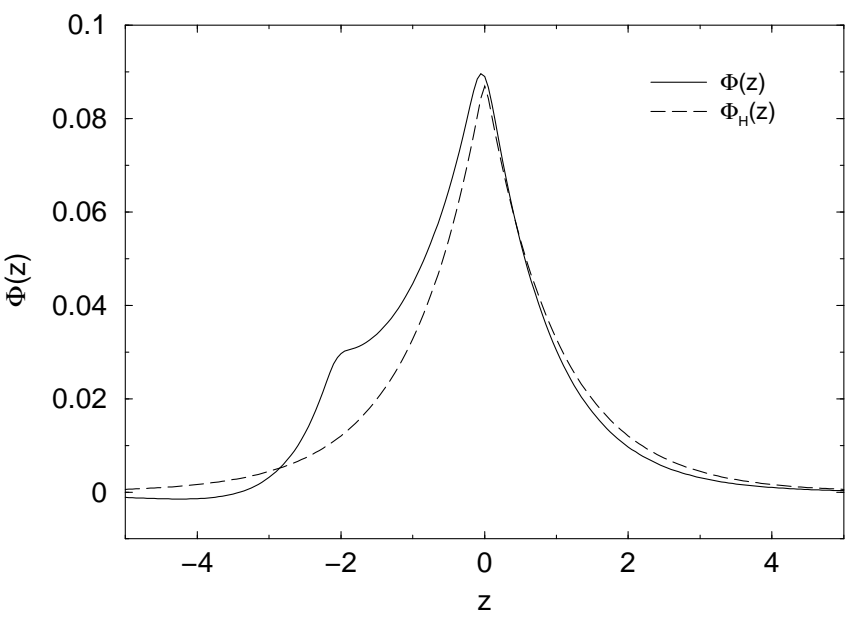

FIG. 6: Atomic orbital obtained for the $\mathrm{H}_{2}^{+}$molecule from a self-consistent solution of the OAO scheme explained in the text and compared to a hydrogenic (1s) orbital $\phi_{H}(z)$. Both curves are plotted along the electronic $z$ axis. In atomic units.

Fig. [6] visualizes the optimized atomic orbital as obtained from Eq. (119) for the $H_{2}^{+}$molecular ion. Compared to a hydrogenic $1 s$ orbital, which is added to the plot in dashed linestyle, we clearly see the anticipated influence of the other nucleus: At a distance of $R=\langle R\rangle \approx 2.2$ a.u., a second peak appears, leading to what we called a polarized orbital. We may view this orbital as being optimized in the sense that it provides the best ground-state solution when used in the expression (124) for $E_{\mathrm{Hc}}^{e n}$. Indeed the results obtained for the $\mathrm{H}_{2}$ and $\mathrm{H}_{2}^{+}$molecules molecule, which are again given in Tables \and tently improve upon the Hartree data. This remains true for the harmonic constant $\omega$, where the deviations found in the Hartree scheme are somewhat reduced within the current approach. Correspondingly, the nuclear densities and potentials are slightly improved, as seen in Figs. [1 - 4 However, the disagreement with the exact curves is still quite large.

To further investigate this point, we have, following Eq. (125), decomposed the conditional potential into its different parts. The results obtained for the $\mathrm{H}_{2}^{+}$molecule are shown in Fig. 7 where $V_{\text {cond,W }}(R)$, Eq. (126), is plotted on the left-hand side and $V_{\text {cond.T }}(R)$, Eq. (127), is plotted on the right-hand side. In addition to the curves obtained from the $\mathrm{OAO}$ and the $\mathrm{BO}$ approach, which again serves as a reference, we added the results calculated from a simple LCAO ansatz using hydrogenic $(1 s)$ atomic orbitals in Eq. (114) instead of the optimized atomic orbitals. The corresponding curve is denoted by $\mathrm{H}(1 s)$-LCAO in Fig. [7] We first observe that the results from the simple LCAO and the optimized OAO scheme are very similar in shape. Yet, the ones obtained from the optimized $\mathrm{OAO}$ are very close to the exact $(\mathrm{BO})$ numbers at the equilibrium internuclear distance $\langle R\rangle$, as clearly visible in Fig. 7 This enables the OAO approach to predict ground-state properties nicely, whereas the simple
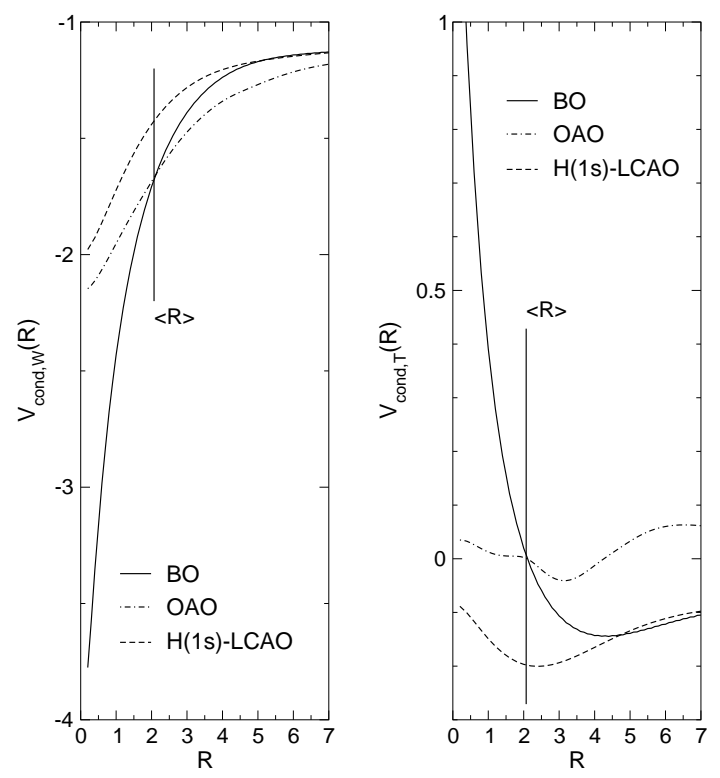

FIG. 7: Contributions to the nuclear conditional potential 125) as obtained from a self-consistent solution of the $M C K S$ $\mathrm{OAO}$ scheme for the $\mathrm{H}_{2}^{+}$molecule. They are compared to the corresponding $B O$ curves and to results provided by a simple LCAO employing hydrogenic (1s) orbitals. In addition, the mean (equilibrium) internuclear distance $\langle R\rangle$ is marked. In atomic units.

LCAO only leads to qualitatively correct results. However, since the optimized OAO potentials are basically shifted (see Fig [6), the $R \rightarrow \infty$ asymptotics, which - by construction - is correctly described by the simple-OAO approach, is incorrect in the present approach. In the unified atom limit, on the other hand, both OAO schemes produce large errors. This is an inherent shortcoming of the OAO approach, which is not set up to satisfy the $R \rightarrow 0$ limit.

In conclusion, the results of the method presented in this section are clearly superior to the ones obtained from the Hartree approach. Since the atomic orbitals are optimized for the molecular ground state, the scheme works nicely for quantities depending mostly on the equilibrium geometry but fails to substantially improve on the Hartree scheme for large internuclear distances. This deficiency will be dealt with in the next section.

\section{Scaling the Atomic Orbitals}

Owing to the successes of the OAO method to describe the bonding region, an ansatz similar to (114) will lay the foundation also for the work presented in this section. However, in order to improve on the shortcomings of the OAO approach, we additionally concentrate on the task of setting up the scheme such that the separated as well as the unified atom limit are correctly reproduced.

The following investigations are again based on the quantity which is considered to be the key quantity to 
approximate, namely the electronic conditional density $\gamma(\mathbf{r} \mid \mathbf{R})$. As above, we start out by decomposing the conditional density into orbital contributions as in Eq. (113) which are approximated by an LCAO-type ansatz

$$
\gamma_{j}(\mathbf{r} \mid \mathbf{R}) \approx \frac{1}{2 \nu_{j}(\mathbf{R})}\left|\phi_{j}^{A}\left(\mathbf{r}_{A}\right)+\phi_{j}^{B}\left(\mathbf{r}_{B}\right)\right|^{2},
$$

and $\mathbf{r}_{A / B}=\mathbf{r} \mp \frac{M_{2 / 1}}{M_{\text {nuc }}} R \mathbf{e}_{z}$ as before. The denominator $\nu_{j}(\mathbf{R})$ arises from the normalization constraint (115) and is given by

$$
\nu_{j}(\mathbf{R})=\frac{1}{2} \int d \mathbf{r}\left|\phi_{j}^{A}\left(\mathbf{r}_{A}\right)+\phi_{j}^{B}\left(\mathbf{r}_{B}\right)\right|^{2} .
$$

As a consequence, the first sumrule 65] for the conditional density is automatically satisfied for any choice of the atomic orbitals.

Up to this point, we just repeated the analysis of Sec. IVC Now, we have to specify the atomic orbitals $\left\{\phi_{j}^{A / B}\right\}$. In the approach presented in the last section, they were determined by optimizing the molecular ground-state configuration. Since these orbitals were further used to describe the large- $R$ behavior of a diatomic molecule, the deviations reported on above were found.

In order to improve on that, we consider the separated atom $(R \rightarrow \infty)$ limit. There, the system consists of two atoms $A$ and $B$, which do not interact among each other. The atoms can thus be described by electronic orbitals, denoted by $\left\{\phi_{\infty, j}^{A / B}\right\}$, which yield the ground-state densities $\rho_{A / B}$ of the fragments. We assume that these orbitals are known, e.g., from an electronic DFT calculation for the single atoms. If the orbitals $\left\{\phi_{\infty, j}^{A / B}\right\}$ were inserted in Eq. (130), we would obtain the simple LCAO scheme which was used for comparison in the last section. Obviously, the bonding effects are not satisfactorily described within such a simple ansatz. In view of the mutual influence of the atoms, we expect the orbitals to change when the atoms approach each other as illustrated by Fig. [ in the last section.

Here, we account for this change by introducing contracted orbitals

$$
\phi_{j}^{A / B}(\mathbf{r}) \equiv \phi_{\lambda, j}^{A / B}(\mathbf{r})=\lambda_{j}^{3 / 2} \phi_{\infty, j}^{A / B}\left(\lambda_{j} \mathbf{r}\right) .
$$

The idea to model bonding effects by such a scaling procedure can be explained in terms of the virial theorem: A decrease of the total energy due to chemical bonding leads to an increase of the kinetic energy and thus to a contraction of the orbitals 54]. Evidently, the size of the contraction depends on the molecular configuration and, in particular, on the internuclear distance. It should therefore be determined self-consistently from the densities which characterize the system, i.e.,

$$
\lambda_{j}=\lambda_{j}[\Gamma, \rho](\mathbf{R}) .
$$

At this point, we already see some benefits of this approach. If, for the equilibrium geometry, a scaling parameter $\lambda>1$ is used, the description of molecular bonding is improved upon the simple LCAO approach. On the other hand, employing $\lambda \rightarrow 1$ for $R \rightarrow \infty$, the large$R$ asymptotics of the conditional density is correctly reproduced. In the following, we will describe a way to calculate the scaling function $\lambda_{j}(\mathbf{R})$ within the MCKS scheme. We start by employing the second sumrule (66). To construct our functional it is assumed that this equation also holds true for its analogue formulated in terms of the orbital densities (as it does in the BO limit),

$$
\rho_{j}(\mathbf{r})=\int d \mathbf{R} \Gamma(\mathbf{R}) \gamma_{j}(\mathbf{r} \mid \mathbf{R}),
$$

where $\rho_{j}(\mathbf{r})=\left|\varphi_{j}(\mathbf{r})\right|^{2}$. If Eq. (134) is satisfied for all $j$, the sumrule (66) is obeyed, too. Employing Eqs. (130) and (132), Eq. (134) is rewritten as

$$
\begin{aligned}
& \rho_{j}(\mathbf{r})=\int d \mathbf{R} \Gamma(\mathbf{R}) \frac{\lambda_{j}^{3}(\mathbf{R})}{2 \nu_{j}(\mathbf{R})} \\
& \times\left|\phi_{\infty, j}^{A}\left(\lambda_{j}(\mathbf{R}) \mathbf{r}_{A}\right)+\phi_{\infty, j}^{B}\left(\lambda_{j}(\mathbf{R}) \mathbf{r}_{B}\right)\right|^{2} .
\end{aligned}
$$

Once the atomic orbitals $\left\{\phi_{\infty, j}^{A / B}\right\}$ are given, the above integral equation determines $\lambda_{j}(\mathbf{R})$ as an (implicit) functional of the nuclear density $\Gamma$ and of the electronic MCKS orbital densities $\rho_{j}$. However, a full solution of this integral equation is rather complicated and will not be attempted in the present approach.

Instead, a simplified scheme appears highly desirable. To this end, we investigate the limits of the scaling function $\lambda_{j}(\mathbf{R})$. As already noted above, we impose the condition that

$$
\lambda_{j}(\mathbf{R}) \stackrel{R \rightarrow \infty}{\longrightarrow} 1,
$$

which guarantees the correct $R \rightarrow \infty$ asymptotic behavior of the conditional density $\gamma(\mathbf{r} \mid \mathbf{R})$. Next, we consider the unified atom limit $R=0$. In that case, the orbital conditional density reads

$$
\gamma_{j}(\mathbf{r} \mid \mathbf{R}=0)=\frac{\lambda_{j}^{3}(0)}{2 \nu_{j}(0)}\left|\phi_{\infty, j}^{A}\left(\lambda_{j}(0) \mathbf{r}\right)+\phi_{\infty, j}^{B}\left(\lambda_{j}(0) \mathbf{r}\right)\right|^{2} .
$$

This quantity should be equal to the electronic orbital density of the unified atom, $\rho_{A+B, j}(\mathbf{r})$. We therefore choose $\lambda_{j}(0)$ such that $\gamma(\mathbf{r} \mid 0)$ most closely resembles $\rho_{A+B, j}(\mathbf{r})$. In other words, $\lambda_{j}(0)$ is obtained from the minimization problem

$$
\min _{\lambda_{j}(0)} \int d \mathbf{r}\left|\gamma_{j}(\mathbf{r} \mid 0)-\rho_{A+B, j}(\mathbf{r})\right|^{2},
$$

where the unified-atom density is assumed to be known.

We illustrate this prescription for $H_{2}^{+}$molecule: In the separated atom limit, the electron is represented by a hydrogenic $(1 s)$ orbital

$$
\phi_{\infty}^{A}(\mathbf{r})=\phi_{\infty}^{A}(\mathbf{r})=\frac{1}{\sqrt{\pi}} \exp (-r),
$$


whereas the density of the unified - in this example: $\mathrm{He}^{+}$ - atom reads

$$
\rho_{A+A}(\mathbf{r}) \equiv \rho_{H e^{+}}(\mathbf{r})=\frac{Z_{H e^{+}}}{\pi} \exp \left(-2 Z_{H e^{+}} r\right) .
$$

From Eq. (138), we immediately obtain

$$
\lambda(0)=Z_{H e^{+}} .
$$

Therefore, $\lambda(0)$ is given by the sum of the charges of the two nuclei. In a more complicated system, we expect the bare nuclear charge $Z_{A+B}$ to be replaced by an effective one. Employing Eq. (141), it is easily seen that the conditional density (130) reproduces the correct unified atom limit.

Furthermore, the small- $R$ behavior of the conditional density is analyzed. This can be done by expanding the electronic Hamiltonian $\hat{H}_{e}$ in powers of $R$, yielding

$$
\hat{H}_{e}=\hat{H}_{A+B}+\frac{M_{1} Z_{2}-M_{2} Z_{1}}{M_{\mathrm{nuc}}} \sum_{j} \frac{z_{j}}{r_{j}^{3}} R+\mathcal{O}\left(R^{2}\right),
$$

where $\hat{H}_{A+B}$ denotes the Hamiltonian of the unified atom. For homonuclear systems, to which we restrict ourselves in all numerical calculations, the first-order correction in Eq. (142) vanishes. From the fact that the electronic density corresponding to (142) basically coincides with $\gamma(\mathbf{r} \mid \mathbf{R})$, we obtain the small- $R(R \rightarrow 0)$ behavior:

$$
\gamma(\mathbf{r} \mid \mathbf{R})=\rho_{A+A}(\mathbf{r})+\mathcal{O}\left(R^{2}\right) .
$$

In view of the limits discussed above, we propose a simple parameterization of the scaling function:

$$
\lambda_{j}(\mathbf{R})=1+\frac{\alpha_{j}}{1+\beta_{j} R^{\gamma}} .
$$

Using such a form, the $R \rightarrow \infty$ limit (136) is fulfilled. The constant $\alpha_{j}$ follows from the unified atom limit, Eq. (138): $\alpha_{j}=\lambda_{j}(0)-1$. The exponent $\gamma$ is chosen such that the model conditional density behaves - for homonuclear molecules - as (143) for $R \rightarrow 0$, leading to $\gamma=2$. We are therefore left with one still unknown coefficient, namely $\beta_{j}$. To determine this constant, we resort to the integral equation (134). Employing additionally quasi-classical nuclei, $\Gamma(\mathbf{R})=\delta(\mathbf{R}-\langle\mathbf{R}\rangle)$, we obtain

$$
\rho_{j}(\mathbf{r})=\gamma_{j}(\mathbf{r} \mid\langle\mathbf{R}\rangle) .
$$

The coefficient $\beta_{j}$ is then obtained self-consistently from fitting the model conditional density to the electronic MCKS orbitals densities:

$$
\min _{\beta_{j}} \int d \mathbf{r}\left|\rho_{j}(\mathbf{r})-\gamma_{j}(\mathbf{r} \mid\langle\mathbf{R}\rangle)\right|^{2} .
$$

Having calculated $\beta_{j}$, we put together all ingredients for the construction of the model conditional density, which is denoted by SAO (scaled atomic orbital) in the following, and finally arrive at

$$
\begin{aligned}
& \gamma^{\mathrm{SAO}}[\Gamma, \rho](\mathbf{r} \mid \mathbf{R})=\sum_{j} \frac{\lambda_{j}^{3}(\mathbf{R})}{2 \nu_{j}(\mathbf{R})} \\
& \times\left|\phi_{\infty, j}^{A}\left(\lambda_{j}(\mathbf{R}) \mathbf{r}_{A}\right)+\phi_{\infty, j}^{B}\left(\lambda_{j}(\mathbf{R}) \mathbf{r}_{B}\right)\right|^{2},
\end{aligned}
$$

with $\lambda_{j}(\mathbf{r})$ given by Eq. (144). Summarizing the above prescription, the parameters in $\lambda_{j}$ are obtained from (i) the atomic orbitals corresponding to the unified and the separate atom limit, which have to be provided as an input, and (ii) self-consistently via Eq. (146) from the MCKS (orbital) densities. Thereby, the conditional density $\gamma^{\mathrm{SAO}}[\Gamma, \rho]$ is an (implicit) functional of the MCKS densities. Moreover, the SAO conditional density now satisfies, by construction, both normalization sumrules (the second sumrule at least in a good approximation), reproduces the correct asymptotic behavior and hence meets all the requirements set up in the beginning of the section.

Having obtained an approximation for the conditional density, we again have to face the problem of the coupling-constant integration in Eq. (62). One possibility to overcome this problem was discussed in Sec. IVC where the Hc energy functional is expressed exclusively in terms of the conditional density at full coupling strength. Employing this expression, Eq. (59), we finally obtain

$$
\begin{aligned}
& E_{\mathrm{Hc}}^{\mathrm{SAO}}[\Gamma, \rho]=\int d \mathbf{R} \Gamma(\mathbf{R}) \\
& \times\left(\int d \mathbf{r} W_{e n}(\mathbf{R}, \mathbf{r}) \gamma^{\mathrm{SAO}}(\mathbf{r} \mid \mathbf{R})+F^{e}\left[\gamma^{\mathrm{SAO}}\right](\mathbf{R})-F^{e}[\rho]\right) .
\end{aligned}
$$

The corresponding nuclear conditional potential is given by

$$
\begin{aligned}
V_{\text {cond }}^{\mathrm{SAO}}(\mathbf{R}) & =V_{\text {cond, } \mathrm{W}}^{\mathrm{SAO}}(\mathbf{R})+V_{\text {cond,T }}^{\mathrm{SAO}}(\mathbf{R}) \\
& +V_{\text {cond, } \mathrm{H}}^{\mathrm{SAO}}(\mathbf{R})+V_{\text {cond,xc }}^{\mathrm{SAO}}(\mathbf{R})
\end{aligned}
$$

with

$$
\begin{aligned}
V_{\text {cond }, \mathrm{W}}^{\mathrm{SAO}}(\mathbf{R}) & =\int d \mathbf{r} W_{e n}(\mathbf{R}, \mathbf{r}) \gamma^{\mathrm{SAO}}(\mathbf{r} \mid \mathbf{R}) \\
V_{\mathrm{cond}, \mathrm{T}}^{\mathrm{SAO}}(\mathbf{R}) & =\frac{1}{8} \sum_{j} \int d \mathbf{r} \frac{\left|\nabla \gamma_{j}^{\mathrm{SAO}}(\mathbf{r} \mid \mathbf{R})\right|^{2}}{\gamma_{j}^{\mathrm{SAO}}(\mathbf{r} \mid \mathbf{R})} \\
& -T_{S, e}[\rho] \\
V_{\mathrm{cond}, \mathrm{H}}^{\mathrm{SAO}}(\mathbf{R}) & =\frac{1}{2} \iint d \mathbf{r} d \mathbf{r}^{\prime} \frac{\gamma^{\mathrm{SAO}}(\mathbf{r} \mid \mathbf{R}) \gamma^{\mathrm{SAO}}\left(\mathbf{r}^{\prime} \mid \mathbf{R}\right)}{\left|\mathbf{r}-\mathbf{r}^{\prime}\right|} \\
& -E_{\mathrm{H}}^{e}[\rho] \\
V_{\text {cond,xc }}^{\mathrm{SAO}}(\mathbf{R}) & =E_{\mathrm{xc}}^{e}\left[\gamma^{\mathrm{SAO}}\right](\mathbf{R})-E_{\mathrm{xc}}^{e}[\rho] .
\end{aligned}
$$

As above, Eq. (149) can be used in the MCKS scheme.

As an interesting aside, we add an alternative approach to calculate the nuclear conditional potential directly from $\gamma^{\lambda=1}(\mathbf{r} \mid \mathbf{R})$. The idea rests on the observation 
that $V_{\text {cond }}(\mathbf{R})$ is practically identical to the lowest energy BO-PES, if non-BO effects are negligible. Employing the Hellmann-Feynman (electrostatic) theorem [55], we obtain

$$
\begin{aligned}
\frac{\partial V_{\text {cond }}(\mathbf{R})}{\partial \mathbf{R}} & \equiv \frac{\partial \epsilon^{\mathrm{BO}}(\mathbf{R})}{\partial \mathbf{R}}=\left\langle\frac{\partial \hat{W}_{\mathrm{en}}}{\partial \mathbf{R}}\right\rangle \\
& =\int d \mathbf{r} \frac{\partial W_{\mathrm{en}}(\mathbf{R}, \mathbf{r})}{\partial \mathbf{R}} \gamma(\mathbf{r} \mid \mathbf{R})
\end{aligned}
$$

Evidently the slope of the nuclear conditional potential is solely determined by the conditional density at full coupling strength and could therefore be evaluated using Eq. (147). Moreover, compared to Eq. (149), the expression (154) seems to be more efficient from a numerical point of view. However, the first approach leading to Eq. (149) proved to be more accurate and will therefore be used in the calculations presented below.

To summarize, the nuclear conditional potential is calculated from Eq. (149) by using the model SAO conditional density (147). As for the OAO approach, we furthermore neglect the nuclear response potential, approximate the electronic potential by the Hartree ansatz, and solve the MCKS scheme self-consistently.

\section{Results}

First of all, we consider the scaling function $\lambda(R)$ obtained from a self-consistent MCKS calculation for the $\mathrm{H}_{2}$ molecule, which is plotted in Fig. 8 By construction, $\lambda(R)$ tends to one for large $R$ such that the dissociation limit is correctly reproduced. For small $R$, on the other hand, we find that $\lambda(0) \approx 1.7$. As expected above, this number is similar to the effective charge one obtains for the $\mathrm{He}$ atom within a simple Hartree-Fock treatment employing hydrogenic orbitals. At the equilibrium distance $\langle R\rangle$, the scaling function acquires a value

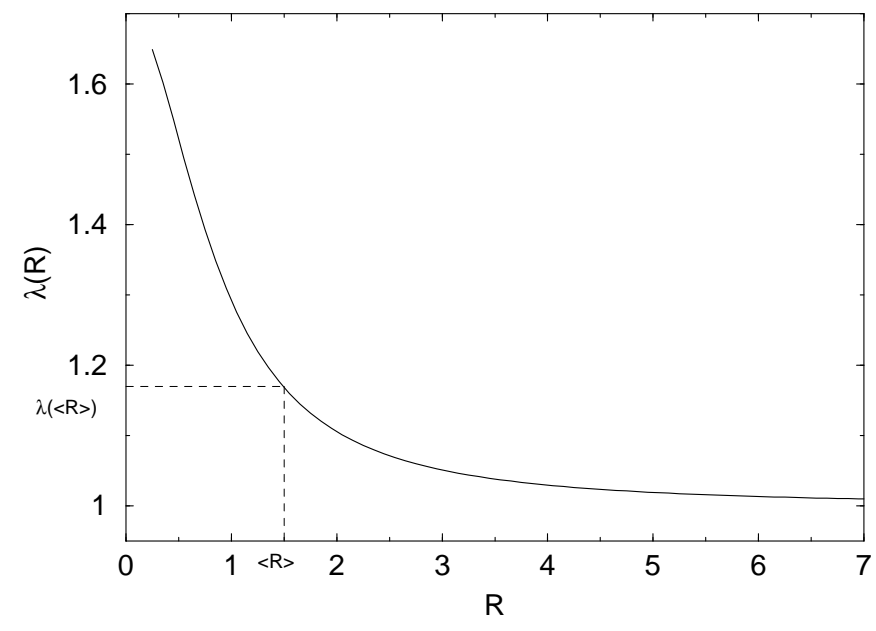

FIG. 8: Scaling function $\lambda(R), E q$. 144), obtained from a self-consistent solution of the MCKS scheme for the $\mathrm{H}_{2}$ molecule. In atomic units.
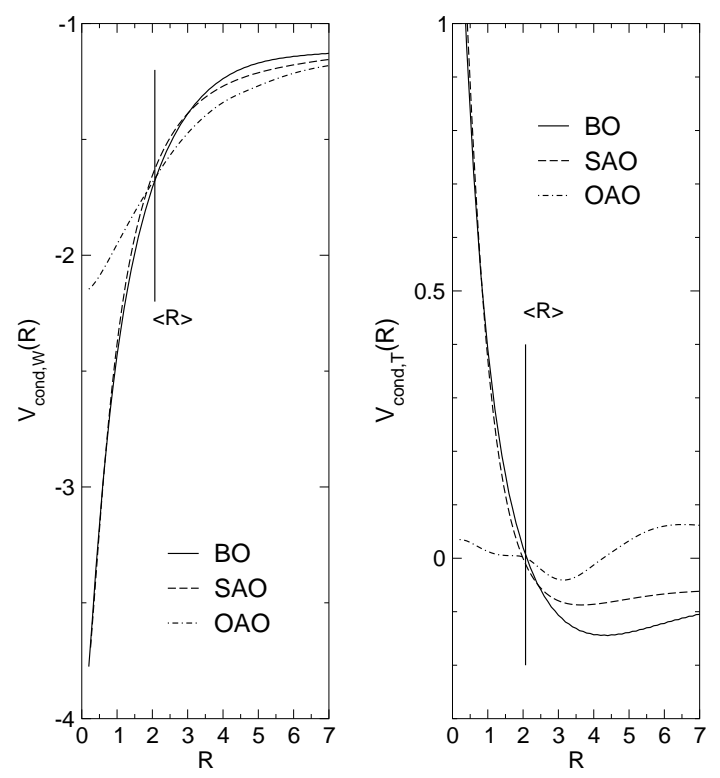

FIG. 9: Comparison of different contributions to the nuclear potential, Eqs. 150) and [151), as obtained for the $\mathrm{H}_{2}^{+}$molecule from the $B O$, the $S A O$, and the OAO method. In atomic units.

of about 1.17 , leading to a contraction of the orbital by this factor. As a consequence, the bonding energy is lowered compared to a simple LCAO ansatz. Indeed, the ground-state energy of the $\mathrm{H}_{2}$ molecule obtained from the $\mathrm{SAO}$ approximation is close to the exact one, as is seen from the last column of Tab. I] The energy improved on the Hartree and on the LCAO data, reducing the error to about $0.5 \%$. We also observe that the $R$ expectation values are slightly overestimated, which can be viewed as a left-over from the simple LCAO method, which generally tends to overestimate the bonding distances. The remaining deviations can be attributed to changes in the orbital like, e.g., the appearance of a second peak as seen in Fig 6] which cannot be accounted for by the simple scaling procedure used in the SAO scheme presented here. This effect seems to be more pronounced for the $\mathrm{H}_{2}^{+}$molecule. From Tab. II we find that especially the ground-state energy is somewhat worse than the results obtained from the other approximations. However, we find a remarkable improvement in the harmonic constant. For both the $\mathrm{H}_{2}^{+}$as well as the $\mathrm{H}_{2}$ molecule, the relative error in $\omega$ is lowered by more than an order of magnitude to about 3\%. Correspondingly, the nuclear densities and potentials are expected to be closer to the exact results, too. From Figs. 1 and 3, we indeed find that the nuclear potentials obtained from the SAO approach are almost indistinguishable from the BO-PES in the asymptotic $(R \rightarrow 0$ and $R \rightarrow \infty)$ regime. Of course, this is hardly surprising, since the correct asymptotic behavior was imposed in the construction of the conditional density. However, this consequently leads to a much improved shape of the nuclear potential, which is obvious from a comparison of the SAO curves to the 
Hartree or LCAO data and is also reflected in the better harmonic constant reported above. Moreover, considering the $\mathrm{H}_{2}$ molecule, the SAO nuclear potential coincides with the BO-PES not only asymptotically, but in the whole $R$ range. This then leads to a nuclear density, plotted in Fig. 4 which nicely agrees with the $\mathrm{BO}$ one. For the $\mathrm{H}_{2}^{+}$molecule, the agreement at intermediate internuclear distances is not as good. From Fig. 2] we find that the nuclear density is slightly shifted to larger $R$. Additionally, the minimum of the SAO nuclear potential is too high, leading to the deviations in the energy already mentioned in the discussion of Tab. III Still, the SAO approach provides the best overall description also for the $\mathrm{H}_{2}^{+}$molecule. In particular, the nuclear density obtained from the SAO approach is closest to the exact one. Furthermore, as seen from Fig 9] where the different contributions to the nuclear conditional potential, Eqs. (150) and (151), are shown, the SAO curves are in satisfactory agreement with the $\mathrm{BO}$ results in the whole range of internuclear distances and thus improve on the OAO method, which only reproduces the correct values around the equilibrium distance. We therefore observe the effect of incorporating the unified as well as separated atom limit into the construction of the Hc energy functional. At this point, we also emphasize the importance to account for the additional contributions which arise from the coupling-constant integration. As is seen from the right-hand side of Fig [9] the $R$-dependence of these terms is significant, and it would not be a good approximation to replace $E_{\mathrm{Hc}}^{e n}$ by $W_{e n}$. In conclusion we find that the SAO approximation to the electron-nuclear correlation functional gives a very good overall description of the BO potential.

\section{CONCLUSIONS}

For a unified quantum mechanical treatment of nuclear and electronic degrees of freedom we extended the traditional density functional method to multicomponent systems. We first discussed the choice of appropriate densities serving as fundamental variables of the theory. It was shown that the usual definition of single-particle densities in terms of inertial coordinates is not well suited for the purpose of this work because such densities, as a consequence of Galilean invariance, are constant for all isolated systems and therefore not characteristic for their internal properties. A suitable set of densities was obtained by defining the electronic density with respect to a coordinate system attached to the nuclear framework whereas the nuclear degrees of freedom were described by the diagonal of the nuclear $N_{n}$-body density matrix. For these fundamental variables the Hohenberg-Kohn theorem and the Kohn-Sham equations were derived and the corresponding density functionals were analyzed in detail. The main new ingredient of the multicomponent theory is the electron-nuclear correlation functional. For this functional several approximations were derived and tested on the $\mathrm{H}_{2}$ and $\mathrm{H}_{2}^{+}$molecules. It was found that the simplest Hartree approximation fails to give a good description of the bonding curve of these molecules. Considerable improvement was obtained using an approximation based on optimized atomic orbitals. This method still had some deficiencies in the large and small bond distance limits. These deficiencies were finally removed using an approximation based on scaled atomic orbitals. Based on this first experience with MCDFT we can say that it presents a promising new approach to study electron-nuclear correlation phenomena beyond the $\mathrm{BO}$ approximation. A promising new field of applications seems the first principle treatment of electron-phonon interactions within a linear response language using a time-dependent extension of the present theory. Another field of future applications will be the study of combined ionization and dissociation dynamics of molecules in strong laser fields. For this case some approximations of similar spirit as discussed in this paper have already been applied succesfully [15, 58].

\section{ACKNOWLEDGEMENTS}

RvL acknowledges support of 'Stichting voor Fundamenteel Onderzoek der Materie (FOM)'.
[1] P.Hohenberg and W.Kohn Phys.Rev.136, B864, (1964)

[2] W.Kohn and L.J.Sham Phys.Rev.140, A1133, (1965)

[3] T. Kreibich and E.K.U. Gross. Phys. Rev. Lett., 86, 2984, (2001).

[4] R.van Leeuwen Phys.Rev. B69, 115110 (2004); 199901(E) (2004).

[5] M. Lüders, M.A.L. Marques, N.N. Lathiotakis, A. Floris, G. Profeta, L. Fast, A. Continenza, S. Massidda, E.K.U. Gross, Phys. Rev. B 72, 024545 (2005).

[6] M.A.L. Marques, M. Lüders, N.N. Lathiotakis, G. Profeta, A. Floris, L. Fast, A. Continenza, E.K.U. Gross, S. Massidda, Phys. Rev. B 72, 024546 (2005).

[7] A. Floris, G. Profeta, N.N. Lathiotakis, M. Lüders,
M.A.L. Marques, C. Franchini, E.K.U. Gross, A. Continenza, and S. Massidda, Phys. Rev. Lett. 94, 037004 (2005).

[8] G. Profeta, C. Franchini, N.N. Lathiotakis, A. Floris, A. Sanna, M.A.L. Marques, M. Lüders, S. Massidda, E.K.U. Gross, A. Continenza, Phys. Rev. Lett. 96, 047003 (2006).

[9] G.D.Mahan Many-Particle Physics Plenum Press (NY), (1990).

[10] K.Hannewald and P.A.Bobbert Phys.Rev. B69, 075212 (2004).

[11] Z.Bačić and J.C.Light Ann.Rev.Phys.Chem. 40, 469 (1989). 
[12] D.Dulić, S.J.van der Molen, T.Kudernac, H.T.Jonkman, J.J.D.de Jong, T.N.Bowden, J.van Esch, B.L.Feringa and B.J.van Wees Phys.Rev.Lett. 91, 207402 (2003).

[13] T.Kreibich Multicomponent Density-Functional Theory for Molecules in Strong Laser Fields, Ph.D. Thesis, University of Würzburg (2000)

[14] T.Kreibich, N.I.Gidopoulos, R.van Leeuwen and E.K.U.Gross in Progress in Theoretical Chemistry and Physics, Vol.14, "The Fundamentals of Electron Density, Density Matrix and Density Functional Theory in Atoms, Molecules and the Solid State" Eds. N.I.Gidopoulos and S.Wilson (Kluwer, Dordrecht) 2003.

[15] T.Kreibich, R.van Leeuwen and E.K.U.Gross, Chem.Phys. 304, 183 (2004).

[16] J.F. Capitani, R.F. Nalewajski, and R.G. Parr. J. Chem. Phys., 76 568, (1982).

[17] H. Goldstein. Classical Mechanics. Addison-Wesley, Reading, 1980.

[18] F.Villars Nucl.Phys. 3, 240 (1957)

[19] F.M.H.Villars and G.Cooper Ann.Phys. 56, 224 (1970)

[20] B.Buck, L.C.Biedenharn, and R.Y.Cusson Nucl.Phys. A 317, 205 (1979)

[21] C.Eckart Phys.Rev. 47, 552 (1935)

[22] E.B.Wilson, J.C.Decius and P.C.Cross, Molecular Vibrations (Dover, New York, 1955)

[23] J.D.Louck and H.W.Galbraith Rev.Mod.Phys.48, 69 (1976)

[24] J.D.Louck J.Mol.Spectrosc. 61, 107 (1976)

[25] P.R. Bunker. Molecular Symmetry and Spectroscopy. Academic Press, New York, 1979.

[26] B.Sutcliffe J.Chem.Soc., Faraday Trans. 89, 2321 (1993)

[27] B.Sutcliffe Adv.Chem.Phys. 114, 1 (2000)

[28] R.G.Littlejohn and M.Reinsch Rev.Mod.Phys. 69, 213 (1997)

[29] R. Schinke. Photodissociation Dynamics. Cambridge University Press, Cambridge, 1993.

[30] L.M. Sander, H.B. Shore, and L.J. Sham. Phys. Rev. Lett. 31 533, (1973).

[31] R.K. Kalia and P. Vashishta. Phys. Rev. B17, 2655, (1978).

[32] N. Gidopoulos. Phys. Rev. B57, 2146, (1998).

[33] X.Lopez, J.M.Ugalde and E.V.Ludeña Eur.Phys.J. D37, 351 (2006)

[34] R.M. Dreizler and E.K.U. Gross. Density Functional Theory. Springer, Berlin, 1990.

[35] M. Levy. Proc. Natl. Acad. Sci. USA 76, 6062, (1979).

[36] G.Chaban, J.O.Jung and R.B.Gerber J.Chem.Phys. 111,
$1823(1999)$

[37] J. Harris and R.O. Jones. J. Phys. F4, 1170, (1974).

[38] D.C. Langreth and J.P. Perdew. Solid State Commun. 17 1425, (1975).

[39] O. Gunnarsson and B.I. Lundqvist. Phys. Rev. B13, 4274, (1976).

[40] G.Hunter Int.J.Quant.Chem.Symp.8, 413 (1974)

[41] G.Hunter Int.J.Quant.Chem.9, 237 (1975)

[42] G.Hunter Int.J.Quant.Chem.17, 133 (1980)

[43] G.Hunter Int.J.Quant.Chem.19, 755 (1981)

[44] J.Czub and L.Wolniewicz Mol.Phys. 36, 1301 (1978)

[45] P.Cassam-Chenä̈ Chem.Phys.Lett. 420, 354 (2006)

[46] N.I.Gidopoulos and E.K.U.Gross cond-mat/0502433 (2005)

[47] M. Berry. Proc. Phys. Soc. (London), A392, 45 (1984).

[48] C. Lanczos. J. Res. Nat. Bur. Stand. 45, 255, (1950).

[49] I.L. Thomas. Phys. Rev. 185, 185, (1969).

[50] I.L. Thomas and H.W. Joy. Phys. Rev. A2, 1200, (1970).

[51] R.T. Sharp and G.K. Horton. Phys. Rev. 90 317, (1953).

[52] J.D. Talman and W.F. Shadwick. Phys. Rev. A14, 36, (1976).

[53] T. Grabo, T. Kreibich, S. Kurth, and E.K.U. Gross. Strong Coulomb Correlations in Electronic Structure: Beyond the Local Density Approximation, pages 203-311. Gordon\&Breach, Tokyo, 1999.

[54] R. van Leeuwen. PhD thesis, Vrije Universiteit, Amsterdam, 1994.

[55] R.P. Feynman. Phys. Rev.56, 340, (1939).

[56] E.H. Lieb. In A. Shimony and H. Feshbach, editors, Physics as Natural Philosophy, page 111. MIT Press, Cambridge, 1982.

[57] A. Shapere and F. Wilczek. Geometric Phases in Physics. World Scientific, Singapore, 1989.

[58] R.van Leeuwen and E.K.U.Gross in "Time-Dependent Density Functional Theory" Eds. M.A.L.Marques, C.A.Ullrich, F.Nogueira, A.Rubio, K.Burke and E.K.U.Gross, Lectures Notes in Physics Vol.706, Springer (Berlin) (2006)

[59] In standard electronic DFT, one can prove that the minimum of $F$ exists 56 .

[60] In the formalism presented here, the Berry-phase effects are assumed to be representable by a scalar nuclear potential. In view of the connection of the Berry phase to a vector potential in the nuclear Schrödinger equation [57], a multicomponent current-density description appears more appropriate to treat these effects. 